\title{
Jovian auroral spectroscopy with FUSE: analysis of self-absorption and implications for electron precipitation
}

\author{
J. Gustin ${ }^{\text {a,* }}$, P.D. Feldman ${ }^{\text {b }}$, J.-C. Gérard ${ }^{\text {a }}$, D. Grodent ${ }^{\text {a }}$, A. Vidal-Madjar ${ }^{\text {c }}$, L. Ben Jaffel ${ }^{\text {, }}$, \\ J.-M. Desert ${ }^{\mathrm{c}}$, H.W. Moos ${ }^{\mathrm{b}}$, D.J. Sahnow ${ }^{\mathrm{b}}$, H.A. Weaver ${ }^{\mathrm{d}}$, B.C. Wolven ${ }^{\mathrm{d}}$, J.M. Ajello ${ }^{\mathrm{e}}$, \\ J.H. Waite ${ }^{\mathrm{f}}$, E. Roueff ${ }^{\mathrm{g}}$, H. Abgrall ${ }^{\mathrm{g}}$ \\ ${ }^{a}$ Laboratoire de Physique Atmosphérique et Planétaire, allée du 6 aout, 17, 4000 Liège, Belgium \\ ${ }^{\mathrm{b}}$ Department of Physics and Astronomy, The Johns Hopkins University, Baltimore, MD 21218, USA \\ c Institut d'Astrophysique de Paris, 75014 Paris, France \\ d Applied Physics Laboratory, Space Department, The Johns Hopkins University, Laurel, MD 20723, USA \\ e Jet Propulsion Laboratory, California Institute of Technology, Pasadena, CA 91109, USA \\ ${ }^{\mathrm{f}}$ Atmospheric, Oceanic and Space Sciences, University of Michigan, Ann Arbor, MI 48109, USA \\ g Observatoire de Paris, Section de Meudon, DAEC and CNRS UMR 8631, 92195 Meudon cedex, France
}

Received 5 September 2003; revised 18 May 2004

Available online 10 August 2004

\begin{abstract}
High-resolution $(\sim 0.22 \AA)$ spectra of the north jovian aurora were obtained in the 905-1180 ̊ window with the Far Ultraviolet Spectroscopic Explorer (FUSE) on October 28, 2000. The FUSE instrument resolves the rotational structure of the $\mathrm{H}_{2}$ spectra and the spectral range allows the study of self-absorption. Below $1100 \AA$, transitions connecting to the $v^{\prime \prime} \leqslant 2$ levels of the $\mathrm{H}_{2}$ ground state are partially or totally absorbed by the overlying $\mathrm{H}_{2}$ molecules. The FUSE spectra provide information on the overlying $\mathrm{H}_{2}$ column and on the vibrational distribution of $\mathrm{H}_{2}$. Transitions from high-energy $\mathrm{H}_{2}$ Rydberg states and treatment of self-absorption are considered in our synthetic spectral generator. We show comparisons between synthetic and observed spectra in the 920-970, 1030-1080, and 1090-1180 Å spectral windows. In a first approach (single-layer model), the synthetic spectra are generated in a thin emitting layer and the emerging photons are absorbed by a layer located above the source. It is found that the parameters of the single-layer model best fitting the three spectral windows are 850 , 800 , and $800 \mathrm{~K}$ respectively for the $\mathrm{H}_{2}$ gas temperature and $1.3 \times 10^{18}, 1.5 \times 10^{20}$, and $1.3 \times 10^{20} \mathrm{~cm}^{-2}$ for the $\mathrm{H}_{2}$ self-absorbing vertical column respectively. Comparison between the $\mathrm{H}_{2}$ column and a 1-D atmospheric model indicates that the short-wavelength FUV auroral emission originates from just above the homopause. This is confirmed by the high $\mathrm{H}_{2}$ rovibrational temperatures, close to those deduced from spectral analyses of $\mathrm{H}_{3}^{+}$auroral emission. In a second approach, the synthetic spectral generator is coupled with a vertically distributed energy degradation model, where the only input is the energy distribution of incoming electrons (multi-layer model). The model that best fits globally the three FUSE spectra is a sum of Maxwellian functions, with characteristic energies ranging from 1 to $100 \mathrm{keV}$, giving rise to an emission peak located at $5 \mu \mathrm{bar}$, that is $\sim 100 \mathrm{~km}$ below the methane homopause. This multi-layer model is also applied to a re-analysis of the Hopkins Ultraviolet Telescope (HUT) auroral spectrum and accounts for the $\mathrm{H}_{2}$ self-absorption as well as the methane absorption. It is found that no additional discrete soft electron precipitation is necessary to fit either the FUSE or the HUT observations.
\end{abstract}

(c) 2004 Elsevier Inc. All rights reserved.

Keywords: Aurora; Jupiter; Spectroscopy; Ultraviolet observations

\section{Introduction}

Ultraviolet spectroscopy of jovian aurorae is a powerful

\footnotetext{
* Corresponding author.

E-mail address: gustin@astro.ulg.ac.be (J. Gustin).
} tool to study the $\mathrm{H}_{2}$ emission produced by the interaction between the atmosphere and the precipitated charged parti- 
cles. The $\mathrm{H}_{2}$ auroral lines are the result of inelastic collisions between primary and secondary electrons with the ambient $\mathrm{H}_{2}$ ground state molecules. The far ultraviolet (FUV) auroral emission is dominated by the $\mathrm{H}_{2}$ Lyman $\left(B^{1} \Sigma_{u}^{+} \rightarrow X^{1} \Sigma_{g}^{+}\right)$ and Werner $\left(C^{1} \Pi_{u} \rightarrow X^{1} \Sigma_{g}^{+}\right)$system bands. Low resolution spectra $(\sim 30 \AA)$ between 875 and $1500 \AA$ were first obtained by the ultraviolet spectrometer (UVS) onboard Voyager (Broadfoot et al., 1979), followed by observations with the International Ultraviolet Explorer (IUE). Yung et al. (1982) compared the IUE spectra between 1200 and $1700 \AA$ with synthetic spectra and established that below $1400 \AA$, the $\mathrm{H}_{2}$ auroral emission is attenuated by methane and other hydrocarbons. They introduced the concept of color ratio, $C=I(1550-1620 \AA) / \mathrm{I}(1230-1300 \AA)$, which is a quantitative measure of the $\mathrm{H}_{2}$ absorption by the overlying hydrocarbon layer. The color ratio is thus related to the altitude of the emission and may be used as an indicator of the penetration depth of the electrons in the atmosphere relative to the hydrocarbon homopause (Livengood et al., 1990; Harris et al., 1996; Gérard et al., 1998). The color ratio is equal to $\sim 1.1$ for an unabsorbed spectrum in units of photons $\mathrm{cm}^{-2} \mathrm{~s}^{-1}$.

Observations with the Goddard High-Resolution Spectrograph (GHRS) on board the Hubble Space Telescope (HST) have been used to determine the effective $\mathrm{CH}_{4}$ column overlying the auroral source and the $\mathrm{H}_{2}$ temperature. The measure of color ratios in different $35-\AA$ bands from GHRS spectra at $\sim 0.5 \AA$ resolution was used by Trafton et al. (1994) and Kim et al. (1997) to derive the $\mathrm{CH}_{4}$ effective column and $\mathrm{H}_{2}$ rotational temperature. They found a $\mathrm{CH}_{4}$ column in the range of $1-7 \times 10^{16} \mathrm{~cm}^{-2}$ and a $\mathrm{H}_{2}$ temperature in the range 300-900 K. Spectra at $\sim 5 \AA$ resolution were also obtained by GHRS between June and September 1996. The $1.74 \times 1.74 \operatorname{arcsec}^{2}$ GHRS aperture was moved to different latitudes and local times, both in the north and south aurora. Dols et al. (2000) fitted these GHRS spectra in the range 1220-1750 $\AA$ and sampled color ratios for various geometric configurations and auroral regions. The synthetic model spectrum they used was coupled with an energy deposition model (Grodent et al., 2001, private communication, 2001) taking into account the vertical dimension of the aurora (temperature, composition and auroral emission distribution with altitude). In this model, a primary electron energy spectrum is injected at the top of the jovian atmosphere. The atmosphere is divided into a number of layers and the model calculates the $\mathrm{H}_{2} \mathrm{UV}$ volume emission rate and the energy spectrum of the degraded electrons as they penetrate deeper into the atmosphere. The densities of the main species $\left(\mathrm{H}_{2}\right.$, $\mathrm{CH}_{4}, \mathrm{C}_{2} \mathrm{H}_{2}$ ) present in the auroral atmosphere are also calculated, assuming vertical turbulent and molecular diffusions in a way consistent with the calculated temperature profile. These output values are used by the synthetic spectrum model to generate an $\mathrm{H}_{2}$ spectrum for each layer. The emerging synthetic spectrum is the sum of the spectra calculated for each layer. Assuming a Maxwellian energy spectrum at the top of the atmosphere, a mean electron energy from 35 to $80 \mathrm{keV}$ was found to best fit the GHRS data. The associ- ated slant $\mathrm{CH}_{4}$ column varied from 2.3 to $70 \times 10^{16} \mathrm{~cm}^{-2}$, respectively.

Spatially resolved spectra obtained with the Space Telescope Imaging Spectrograph (STIS) were employed to characterize the interaction between the precipitated electrons and the atmosphere. Gustin et al. (2002) used two pairs of 50- $\AA$ wide G140M spectra at $\sim 1 \AA$ resolution to determine the $\mathrm{CH}_{4}$ column, $\mathrm{H}_{2}$ temperature and mean energy of the precipitated electrons. They applied the synthetic spectral generator coupled with the one-dimensional model of (Grodent et al., 2001, private communication, 2001), as described in Dols et al. (2000). The coupled model that best fitted the observed $\mathrm{H}_{2}$ line intensity distribution had a mean electron energy of $\sim 80 \mathrm{keV}$. Gérard et al. (2003) analyzed time tagged STIS spectra of the high latitude auroral emission. They showed that the color ratio associated with these polar emissions is consistent with mean electron energy precipitation ranging from 40 to $120 \mathrm{keV}$, a range of values close to those deduced for the auroral oval.

While the long-wavelength far ultraviolet auroral emission is dominated by the $\mathrm{H}_{2}$ Lyman and Werner bands and the Lyman continuum, the short-wavelength FUV spectrum between 900 and $1200 \AA$ includes the higher energetic Rydberg transitions $\left(B^{\prime 1} \Sigma_{u}^{+} \rightarrow X^{1} \Sigma_{g}^{+}, D^{1} \Pi_{u} \rightarrow X^{1} \Sigma_{g}^{+}\right.$, $B^{\prime \prime 1} \Sigma_{u}^{+} \rightarrow X^{1} \Sigma_{g}^{+}$, and $\left.D^{\prime 1} \Pi_{u} \rightarrow X^{1} \Sigma_{g}^{+}\right)$. The relative intensity distribution of the lines mainly depends on the $\mathrm{H}_{2}$ gas temperature profile but below $\sim 1200 \AA$, the transitions connecting to the $v^{\prime \prime}=0,1,2$ levels are totally or partially absorbed by the overlying column of $\mathrm{H}_{2}$. This self-absorption process provides a measure of the $\mathrm{H}_{2}$ overlying column (i.e., the altitude of the FUV emission) and of the population of the ground-state vibrational levels. Analyzing the Voyager UVS dataset, Shemansky and Ajello (1983) and Shemansky et al. (1985) first pointed out the strong attenuation of the $\mathrm{H}_{2}$ bands due to self-absorption below $1000 \AA$. FUV and EUV spectra of Jupiter aurora were collected by the Hopkins Ultraviolet Telescope (HUT) and by the Galileo Extreme U1traviolet Spectrometer (EUVS), in the period from 1995 to 1997. Analysis of the 3 - $\AA$ resolution HUT auroral spectrum in the range 850-1700 $\AA$ provided quantitative results about self-absorption (Wolven and Feldman, 1998). Wolven and Feldman (1998) developed a synthetic $\mathrm{H}_{2}$ model taking into account the effect of self-absorption and found that the transitions connecting to $v^{\prime \prime}=0$ were almost totally absent in the emergent spectrum, while the lines connecting to $v^{\prime \prime}=1$ and $v^{\prime \prime}=2$ were strongly attenuated. An $\mathrm{H}_{2}$ vertical column of $1.5 \times 10^{20} \mathrm{~cm}^{-2}$ and a column-averaged temperature of $900 \mathrm{~K}$ provided the best fit between the data and their model. The $\sim 30 \AA$ resolution Galileo EUVS data between 540$1280 \AA$ and the HUT auroral spectrum were analyzed with synthetic spectra by Ajello et al. (1998). They modeled the EUV and FUV emissions by a primary electron distribution function composed of Maxwellian and Kappa distributions over the electron energy range of soft (20-200 eV) and hard (5-100 keV) electrons. They concluded that the EUV and FUV emissions are distributed throughout the thermosphere, 
from the homopause to the exobase, near $2000 \mathrm{~km}$. Soft electrons $(27 \mathrm{eV})$ were needed to enhance the EUV emission in their synthetic model, while the hard electrons contributed to the main emission peaking near $245 \mathrm{~km}$ (Ingersoll et al., 1998; Vasavada et al., 1999).

To further investigate the relation between the population of the $\mathrm{H}_{2}$ rovibrational levels in the auroral atmosphere and the depth of the energy deposition, we describe in this study a set of high-resolution spectra $(\sim 0.22 \AA)$ taken by the Far Ultraviolet Spectroscopic Explorer (FUSE) spacecraft. This resolution allows a detailed study of the $\mathrm{H}_{2}$ rotational structure, which was not possible with previous instruments in this spectral range. After an upgrade of the synthetic spectral generator (addition of the Rydberg electronic states and numerical treatment of self-absorption), we present comparisons between observed spectra and model. Two methods have been considered to generate the synthetic spectra. The first approach assumes that the aurora is produced in a single narrow emitting layer (single-layer model), characterized by a mean rovibrational temperature. This layer is overlaid by an absorbing layer of $\mathrm{CH}_{4}$ and $\mathrm{H}_{2}$. Since the three parameters $\left(\mathrm{H}_{2}\right.$ column, $\mathrm{CH}_{4}$ column and $\mathrm{H}_{2}$ temperature) are linked and influence each other, the numerical model performs a series of nested loops with temperature, the $\mathrm{H}_{2}$ column and the $\mathrm{CH}_{4}$ column as variable parameters for each loop. Each resulting synthetic spectrum is compared with the data and the synthetic spectrum that minimizes the chisquares is considered as the best fit. In the second method (multi-layer model), the synthetic spectra are produced by the spectral generator coupled with an energy degradation model, as in Dols et al. (2000) and Gustin et al. (2002). Series of electron energy distributions are considered, and the emerging synthetic spectrum for each case is compared with the data. The model that gives the best fit is believed to provide an accurate estimate of the incident electron energy flux and of the auroral atmosphere at the time of the observa- tions. The model parameters leading to the best fit (single layer approach) and the specifications of the best 1-D model (multi-layer approach) are discussed and the implications on the auroral characteristics are considered.

\section{Observations}

Spectra of Jupiter's north auroral region in the wavelength range 905 to $1180 \AA$ were obtained by FUSE on 2000 October 28, starting at 15:10:39 UT. An accumulated exposure of 9708 seconds was obtained by adding 3 spectra obtained during contiguous orbits. One third of the total exposure was acquired during orbit day (not in the Earth's shadow). The spectra were obtained using the low resolution $\left(30^{\prime \prime} \times 30^{\prime \prime}\right)$ LWRS aperture. The center of the LWRS aperture was placed on the central meridian longitude (CML) at $60^{\circ} \mathrm{N}$ at the beginning of each exposure. The motion of Jupiter on the sky was then tracked during the exposure. The CML varied from $80^{\circ}$ at the beginning of the first exposure to $230^{\circ}$ at the end of the third exposure, as illustrated by the HST images shown in Fig. 1. FUSE does not provide any spatial information on the distribution of the emission within the aperture but it is apparent from Fig. 1 that the jovian aurora underfills the FUSE aperture and also moves within the aperture over the time span of the observation.

Proper tracking was verified by the relative constancy of the integrated count rate with time although there was a slow change in this rate as the auroral oval rotated through the field of view, and there were occasional $20 \%$ enhancements in the count rate with durations of a few hundred seconds. These will not be discussed further here. At the time of the observation, Jupiter's heliocentric distance was $5.03 \mathrm{AU}$ and its geocentric distance was 4.17 AU. Details of the FUSE instrumentation have been given by Moos et al. (2000) and Sahnow et al. (2000). Due to the extended but

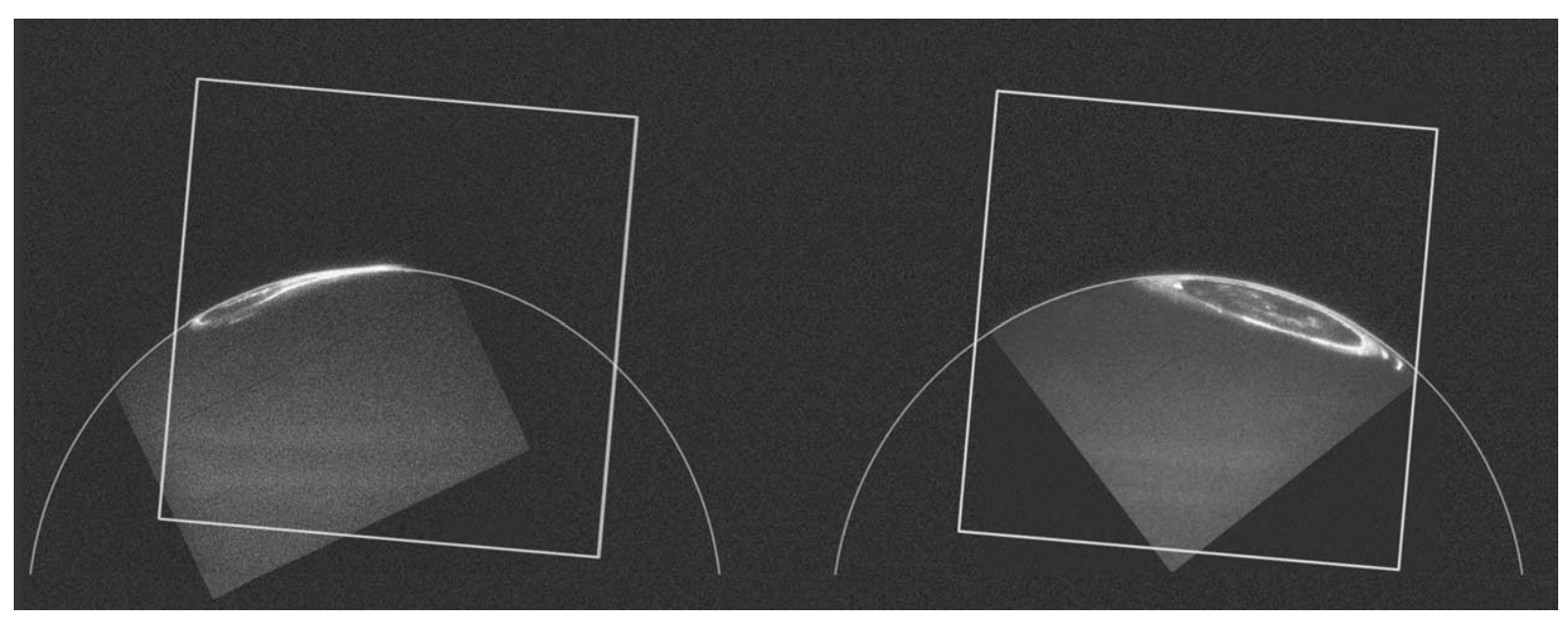

Fig. 1. Field of view of the FUSE LWRS aperture at the beginning and at the end of the observation, using two (unrelated) HST/STIS images with proper CMLs. Since the full aurora fills the aperture, the FUSE spectra consist of a mixture of features from the different regions characterizing the aurora (main oval, polar cap, limb), from the jovian disk, and features from the Io torus that cross the line of sight of the observation. The Io footprint was not visible at the time of the observations. 

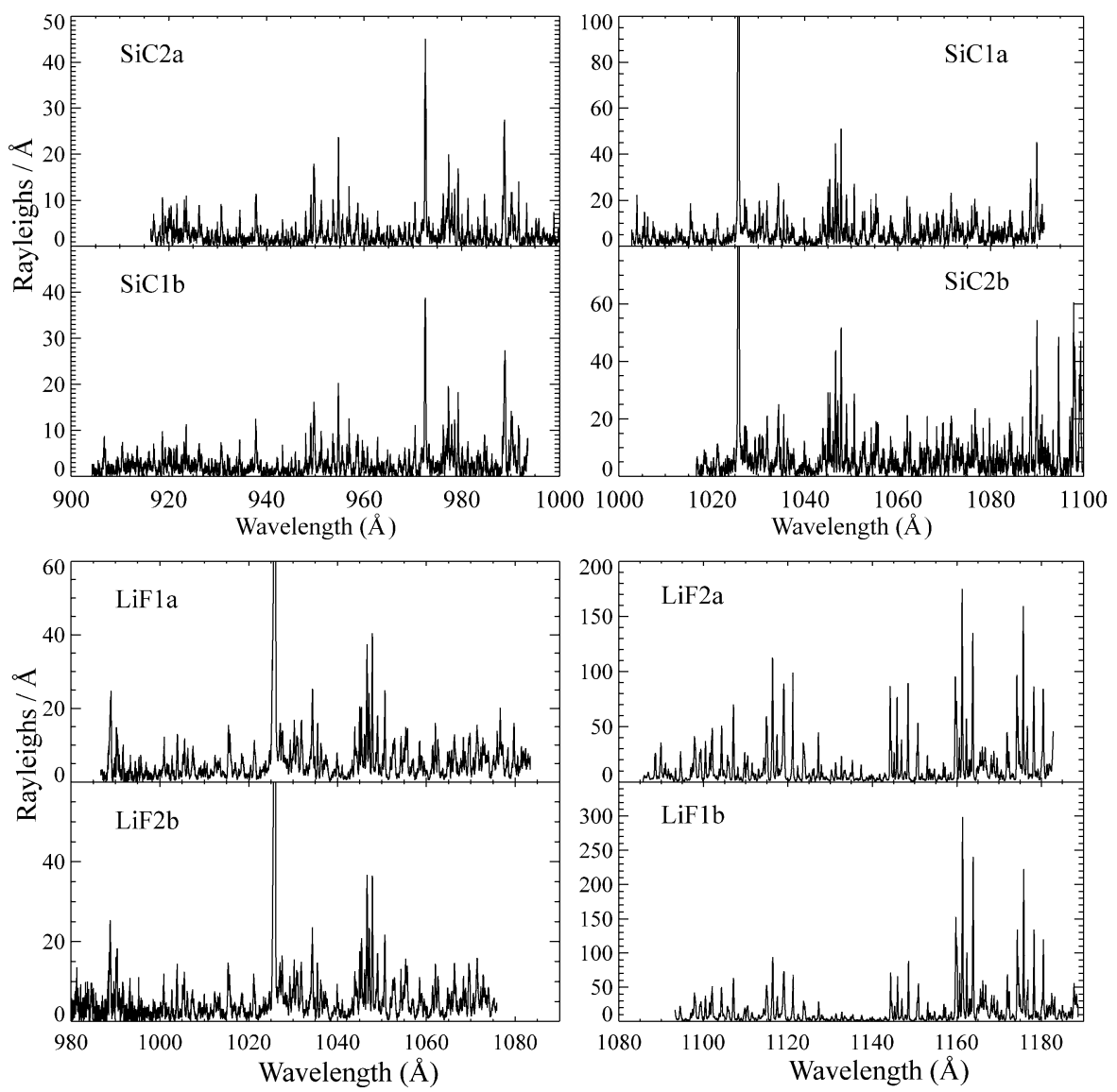

Fig. 2. Overview of the FUSE dataset obtained on 2000 October 28, smoothed over 7 data points.

finite size of the aurora and Doppler broadening caused by its motion within the aperture during the exposure, the effective spectral resolution is $0.22 \AA$. The data presented here were reprocessed with the FUSE pipeline, calfuse, version 2.2.1. The data provided by the three orbits were co-added together, and the extracted fluxes were converted to average brightness (in rayleighs $\AA^{-1}$ ) in the $30^{\prime \prime} \times 30^{\prime \prime}$ aperture.

FUSE consists of four separate telescope/spectrograph assemblies, two employing lithium fluoride coatings (LiF) and two using silicon carbide (SiC). Each of four separate detectors (denoted 1a, 1b, 2a, and 2b) simultaneously records two spectra, one each from a $\mathrm{LiF}$ and a $\mathrm{SiC}$ channel spanning a given wavelength interval, giving a total of eight separate raw spectra. These are shown in Fig. 2. The spectrum is dominated by electron impact excited emissions of $\mathrm{H}_{2}$ and $\mathrm{H}$, but also includes foreground Io torus emissions, terrestrial airglow (mainly atomic oxygen and hydrogen), and jovian disk emission from solar induced fluorescence of $\mathrm{H}_{2}$, principally from Lyman $\beta$ and O VI at $1032 \AA$. The first two contaminants were also seen in the lower resolution ( $\sim 3 \AA$ ) observations of jovian airglow by the Hopkins Ultraviolet Telescope (Feldman et al., 1993). At the higher resolution of FUSE they are easy to separate from the disk emissions. FUSE spectra of the jovian airglow were recently obtained with the medium resolution $\left(4^{\prime \prime} \times 20^{\prime \prime}\right)$ MDRS aperture. Taking into account the resolution of the MDRS com- pared to the LWRS and assuming that the jovian airglow filled $50 \%$ of the LWRS aperture during the observations analyzed here (Fig. 1), it is found that the airglow contribution to the observed auroral spectrum is about $\sim 15 \%$. Additional observations of jovian aurora, both north and south, were made on 2000 December 31 and 2001 January 13, near the time of the Cassini fly-by of Jupiter. These spectra are qualitatively similar to those obtained in October 2000 but have somewhat lower count rates, so we will concentrate our initial analysis on the early data.

\section{The spectral model}

\subsection{Spectral model concept}

The $\mathrm{H}_{2}$ synthetic spectral generator simulates the effects of the impact of the precipitated electrons on the ambient $\mathrm{H}_{2}$ auroral atmosphere and the resulting $\mathrm{H}_{2}$ FUV spectrum. The synthetic spectra, described and used by Dols et al. (2000) and Gustin et al. (2002), included $\mathrm{H}_{2}$ lines belonging to the Lyman $\left(B^{1} \Sigma_{u}^{+} \rightarrow X^{1} \Sigma_{g}^{+}\right)$and Werner $\left(C^{1} \Pi_{u} \rightarrow X^{1} \Sigma_{g}^{+}\right)$ transitions. The population of the $\mathrm{B}$ and $\mathrm{C}$ states is controlled by the direct primary and secondary electron impact on ground state $\mathrm{H}_{2}$ molecules (assumed to be in thermodynamic equilibrium). It is also assumed that the populating of 
the $\mathrm{B}$ and $\mathrm{C}$ electronic states by electron impacts follows the same rules as for radiative transitions (Born approximation). The line transition probabilities by Abgrall et al. (1993a, 1993b) are used to calculate the electron impact excitation cross-section. The cross-section dependence on electron energy uses the formulation of Shemansky et al. (1985) with the updated coefficients by Liu et al. (1998). The population of the B state by the $\left(E, F^{1} \Sigma_{g}^{+} \rightarrow B^{1} \Sigma_{u}^{+}\right)$cascade process is also included in the code. Cascades transitions from $G, K^{1} \Sigma_{g}^{+}$, and $H^{1} \Sigma_{g}^{+}$states are neglected (Dziczek et al., 2000). The $(E, F \rightarrow B)$ cascade terms are calculated from Shemansky et al. (1985) for the vibrational levels and from the Hönl-London factors for the rotational population. The total cascade cross-section is updated to the value found in Dziczek et al. (2000). The Lyman bands mentioned hereafter include both the direct and cascade contributions.

\subsection{Updates and new features}

\subsubsection{Addition of the Rydberg transitions}

Spectra at wavelengths longward of Lyman $\alpha$ (Ly $\alpha)$ at $1216 \AA$ can be described and reproduced with models including only the Lyman and Werner bands (Clarke et al., 1994; Kim et al. 1997; Trafton et al., 1998; Dols et al., 2000; Gustin et al., 2002). Shortward of Ly $\alpha$, the lines belonging to the Rydberg bands need to be considered to provide a detailed description of the $\mathrm{H}_{2}$ spectra. In order to study the short-wavelength FUV domain, the $B^{\prime 1} \Sigma_{u}^{+}, D^{1} \Pi_{u}, B^{\prime \prime 1} \Sigma_{u}^{+}$, and $D^{\prime 1} \Pi_{u}$ electronic states of $\mathrm{H}_{2}$ were added to our spectral model. As for the $\mathrm{B}$ and $\mathrm{C}$ states, the population of the $B^{\prime}, D, B^{\prime \prime}$, and $D^{\prime}$ states by electron impact is determined by the Shemansky formulation. The line transition probabilities of $B^{\prime}-X$ and $D-X$ have been calculated by Abgrall et al. (1994) and the corresponding dissociation probabilities are reported in Abgrall et al. (2000). New calculations have been performed for the $B^{\prime \prime}$ and $D^{\prime}$ states by extending the coupling between excited electronic states of $\mathrm{H}_{2}$. The electronic potentials and radial couplings of $B^{\prime \prime}$ and $D^{\prime}$ are taken respectively from Reinhold et al. (1999) and Spielfiedel (2003). Details of the calculations can be found in Abgrall et al. (2004) in relation with experimental studies of the electron impact emission spectrum of $\mathrm{H}_{2}$. Figure 3 shows the contribution of each band system to the $\mathrm{H}_{2}$ emission, calculated for $100 \mathrm{eV}$ electron impact with $\mathrm{H}_{2}$ molecules at $500 \mathrm{~K}$. It is seen that the Lyman and Werner bands, which are the only source of $\mathrm{H}_{2}$ lines longward of Ly $\alpha$, still remain significant in the 950-1200 $\AA$ domain. Below $950 \AA$, the Rydberg bands dominate the spectrum while the $B-X$ and $C-X$ transitions have minor contributions. Note that Fig. 3 shows the $\mathrm{H}_{2}$ lines without the effect of self-absorption. The relative contribution of each band system will be altered by self-absorption: below $1000 \AA$, most of the Lyman and Werner emission will be self-absorbed and will become secondary compared to the Rydberg bands emission. A good way to validate the $\mathrm{H}_{2}$ synthetic spectral generator is to compare the model with a laboratory spectrum obtained under controlled conditions. A synthetic spectrum was compared with the high-resolution $(\sim 0.1 \AA)$ electron-impact spectrum described in (Jonin et al., 2000). This laboratory spectrum was produced with electrons of $100 \mathrm{eV}$ at a pressure of $1.2 \times 10^{-5}$ torr, at room temperature $(300 \mathrm{~K})$. The comparison with a synthetic spectrum generated at $300 \mathrm{~K}$ with $100 \mathrm{eV}$ electrons is presented in Fig. 4. The model is in excellent agreement with the laboratory spectrum in these conditions of negligible self-absorption. Note that the model does not include the lines belonging to the H Lyman series (Ly $\alpha$, Ly $\beta$, Ly $\gamma$, Ly $\delta$, Ly $\varepsilon$ ).

\subsubsection{Self-absorption modeling}

The formulation used to model the $\mathrm{H}_{2}$ self-absorption is based on Wolven and Feldman (1998). They use a method where the branching ratio of each individual line from a given $\left(v^{\prime}, j^{\prime}\right)$ level is multiplied by a transmission factor defined by

$T\left(\tau_{s}\right)=\frac{\int_{-\infty}^{+\infty}\left(1-e^{\left(-\tau_{s} \psi(x)\right)}\right) d x}{\int_{-\infty}^{+\infty} \tau_{s} \psi(x) d x}$,

where $\tau_{s}$ is the slant optical depth at line center, $\psi(x)$ is the line profile of the transition. In this expression:

$\tau_{\lambda}=n \sigma_{\lambda} S$

where $n$ is the relative population of absorbing $\mathrm{H}_{2}$ molecules in the $\left(v^{\prime \prime}, j^{\prime \prime}\right)$ state, assumed to follow a Boltzmann distribution, $S$ is the $\mathrm{H}_{2}$ column, and $\sigma_{\lambda}$ is the line absorption cross-section.

Our new code that includes self-absorption was validated using a laboratory spectrum obtained at high pressure $\left(2.2 \times 10^{-4}\right.$ torr, corresponding to an $\mathrm{H}_{2}$ column of $\sim 1 \times$ $10^{15} \mathrm{~cm}^{-2}$ ) for which self-absorption is present (Ajello, personal communication). The comparison between the (selfabsorbed) laboratory spectrum and a synthetic spectrum calculated with the same parameters is shown in Fig. 5. For better legibility, the fits displayed in Figs. 4 and 5 are convolved with a Gaussian function of $0.3 \AA$ FWHM. As for the low pressure laboratory spectrum, the agreement between the model and the laboratory spectrum is excellent. Comparing Figs. 4 and 5, the effect of self absorption is clearly seen below $1030 \AA$, where all the spectral lines are strongly attenuated. A straightforward comparison between the complete $(\mathrm{EUV}+\mathrm{FUV}) \mathrm{H}_{2}$ synthetic spectrum with and without self-absorption (Fig. 6) illustrates the intensity redistribution of the $\mathrm{H}_{2}$ lines. Strong absorption below $1100 \AA$ is clearly seen in Fig. 6. The energy absorbed at short wavelengths is redistributed at longer wavelengths by self-absorption. Since self-absorption is a redistribution of the line intensity rather than an effective absorption, the total intensity of the UV spectrum must be conserved. Tests were made on self-absorbed/unabsorbed pairs of synthetic spectra and the difference between the total line intensity does not exceed $1 \%$. Since photons from the Lyman continuum are in the wavelength range $1200-1750 \AA$, they cannot be absorbed by 


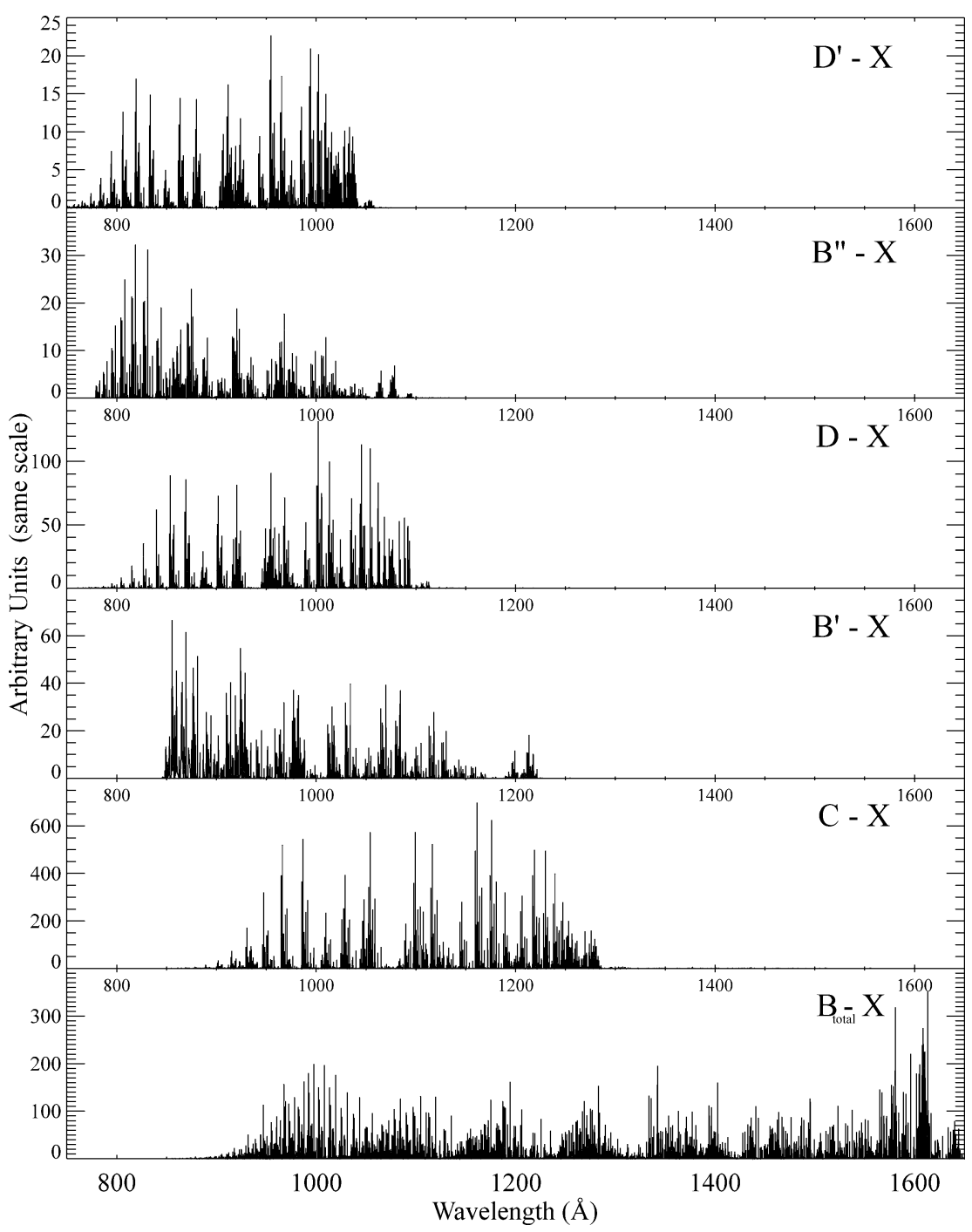

Fig. 3. Contribution of the $\mathrm{H}_{2}$ electronic states included in the synthetic spectral generator. The Rydberg states $\left(B^{\prime}, D, B^{\prime \prime}, D^{\prime}\right)$ were recently included to study the EUV and short-wavelength FUV observed jovian spectra. The $B-X$ spectrum contains both the direct and $E, F$ cascade contributions. This model was obtained at $500 \mathrm{~K}$ with $100 \mathrm{eV}$ electrons.

$\mathrm{H}_{2}$ through a radiative process. Accordingly, the continuum photons are not influenced by self-absorption.

\section{Analysis-results}

We now examine three spectral regions of the FUSE spectra obtained on October 28, 2000. In order to obtain the best signal to noise ratio, the spectra obtained during the three contiguous orbits where summed to a total exposure of 9708 seconds. It should be noted that since the FUSE aperture samples the entire auroral region, it is likely that the resulting spectra account for auroral regions characterized by different temperature, $\mathrm{H}_{2}$ column and color ratio. This mixing effect is further increased by the continuous variation of the view angle and the line of sight of the observation during the exposure. The view angle is defined as the angle between the local vertical and the FUSE satellite.

\subsection{One layer analysis}

In a first approach, we used a simple model to analyze the FUSE data. The synthetic spectral generator described in the previous paragraph is driven by two parameters: the $\mathrm{H}_{2}$ rovibrational temperature (that we will refer to as Tem), which controls the population of the vibrational and rotational levels of the ground-state $\mathrm{H}_{2}$ emitting molecules, and the energy of the precipitated electrons which populate the excited $\mathrm{H}_{2}$ electronic states through inelastic collisions. The emitted EUV and FUV photons can interact with the atmosphere in two ways:

(1) Methane is an active absorber in the $900-1200 \AA$ domain. The shape of the $\mathrm{CH}_{4}$ cross-section absorption varies with wavelength and thus the $\mathrm{CH}_{4}$ column can influence the relative intensity between different spectral regions of a given spectrum. 

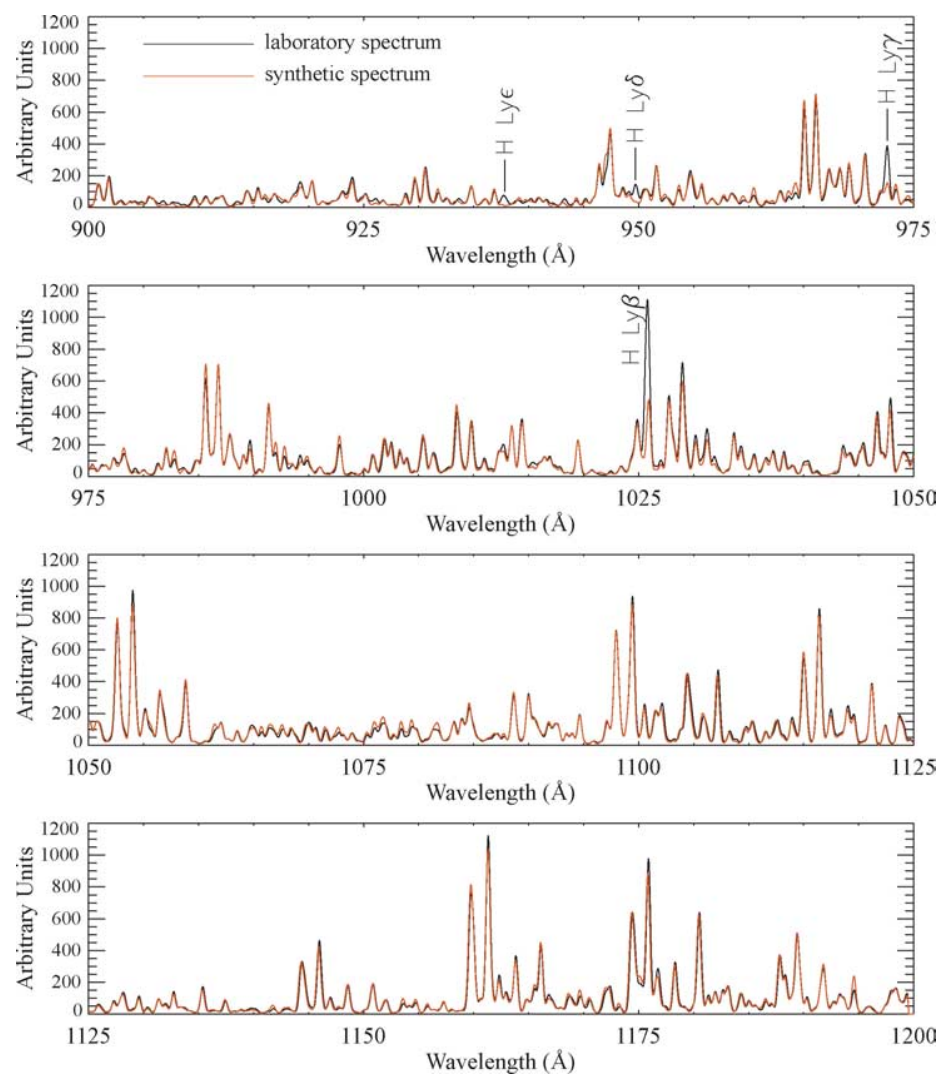

Fig. 4. Comparison between a $\mathrm{H}_{2}$ laboratory spectrum obtained at $300 \mathrm{~K}$ with $100 \mathrm{eV}$ electrons and a synthetic spectrum calculated with the same parameters. The H Lyman series lines are not included in the code.
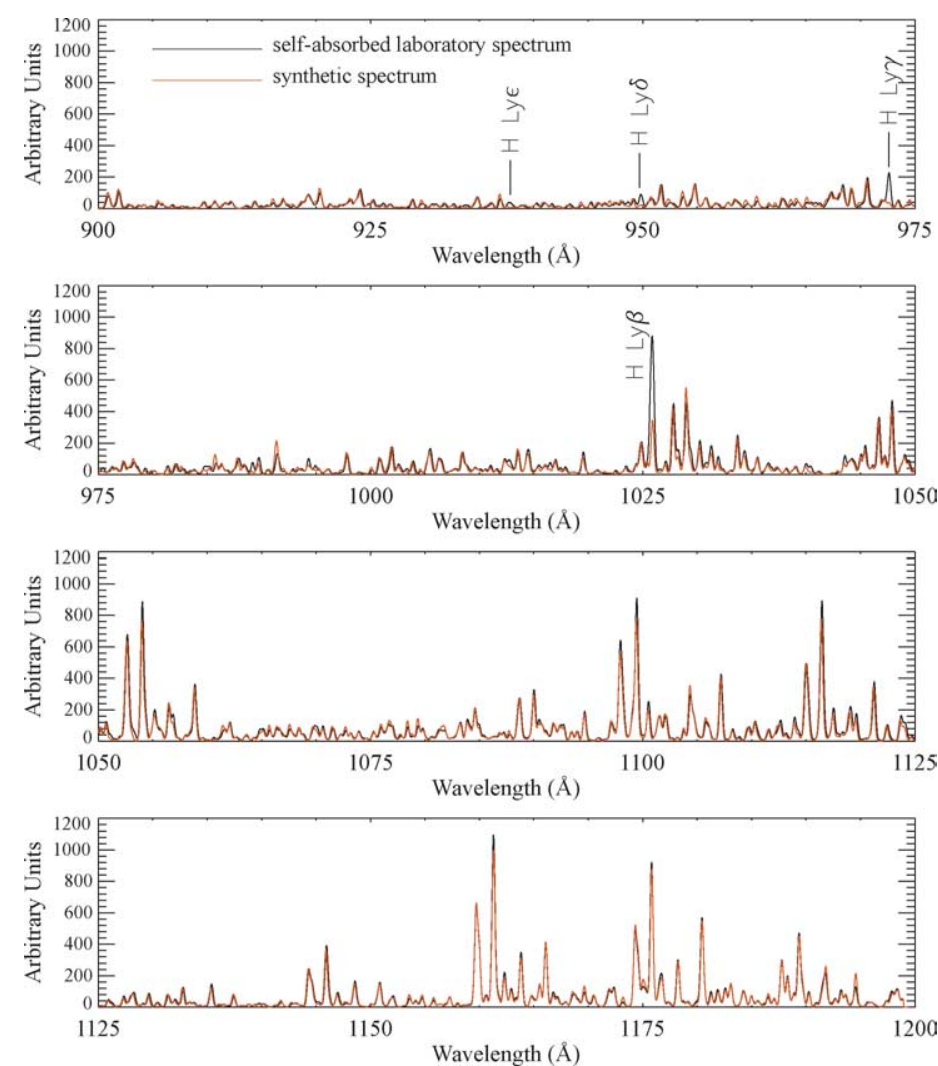

Fig. 5. Comparison between a high-pressure $\mathrm{H}_{2}$ laboratory spectrum (self-absorbed) and a self-absorbed synthetic spectrum with an $\mathrm{H}_{2}$ absorbing column of $\sim 3 \times 10^{15} \mathrm{~cm}^{-2}(T=300 \mathrm{~K}$, $E_{e}=100 \mathrm{eV}$ ). Self-absorption is clearly seen for lines shortward of $1050 \AA$ 
(2) $\mathrm{H}_{2}$ (mainly in the vibrational ground state) can also absorb photons, which results in an increase of the population of the $\mathrm{H}_{2}$ upper electronic states. The de-excitation process by radiative transitions that follows (fluorescence) produces photons that are less energetic than the photons that were initially absorbed. This degradation process changes the intensity distribution of the $\mathrm{H}_{2}$ lines in the auroral spectra, as illustrated before.

These two absorption processes are also implemented in the code where they are controlled by the $\mathrm{CH}_{4}$ column and the $\mathrm{H}_{2}$ column, respectively. The temperature used to calculate the population of rovibrational levels of the $\mathrm{H}_{2}$ absorbing molecules (that we will refer to as $T s a$ ) is assumed to be equal to Tem. In order to reduce the number of parameters, the effective energy of the $\mathrm{H}_{2}$-exciting electrons was set to $100 \mathrm{eV}$. The actual energy spectrum of electrons is distributed between the primary high energy component and the secondary (and tertiary) electrons, produced by ionization of $\mathrm{H}_{2}$ by electron impact. The $100 \mathrm{eV}$ value is used as a mean value for the $\mathrm{H}_{2}$ excitation process, found after folding the calculated electron energy distribution with the total singlet state excitation cross-section. Since the three remaining parameters (Tem, $\mathrm{H}_{2}$ and $\mathrm{CH}_{4}$ columns) are interdependent, we vary one parameter with the other two being held fixed, using three nested loops. The first step of the study is to select a range of Tem, $\mathrm{H}_{2}$ column and $\mathrm{CH}_{4}$ column that are guessed to be reasonable. A first series of synthetic spectra is generated and each model is compared to the observed spectrum. Each comparison is characterized by a chi-square $\left(\chi^{2}\right)$. The second step is to use the parameters giving the minimum $\chi^{2}$ as central value to restrict the range of parameters in a series of new loops. This iterative process continues until a good fit is obtained and may not be significantly improved. In practice the number of models generated in each step can vary from 20 to 1500 , depending on the parameters range for each loop. The best parameters found with this method are summarized in Table 1, where the $\mathrm{CH}_{4}$ and $\mathrm{H}_{2}$ columns were converted into vertical values, assuming a mean view angle of $78^{\circ}$.

\subsubsection{0-970 A window-SiC2a}

This first spectral window contains the highest energy transitions and is thus composed of a large number of lines
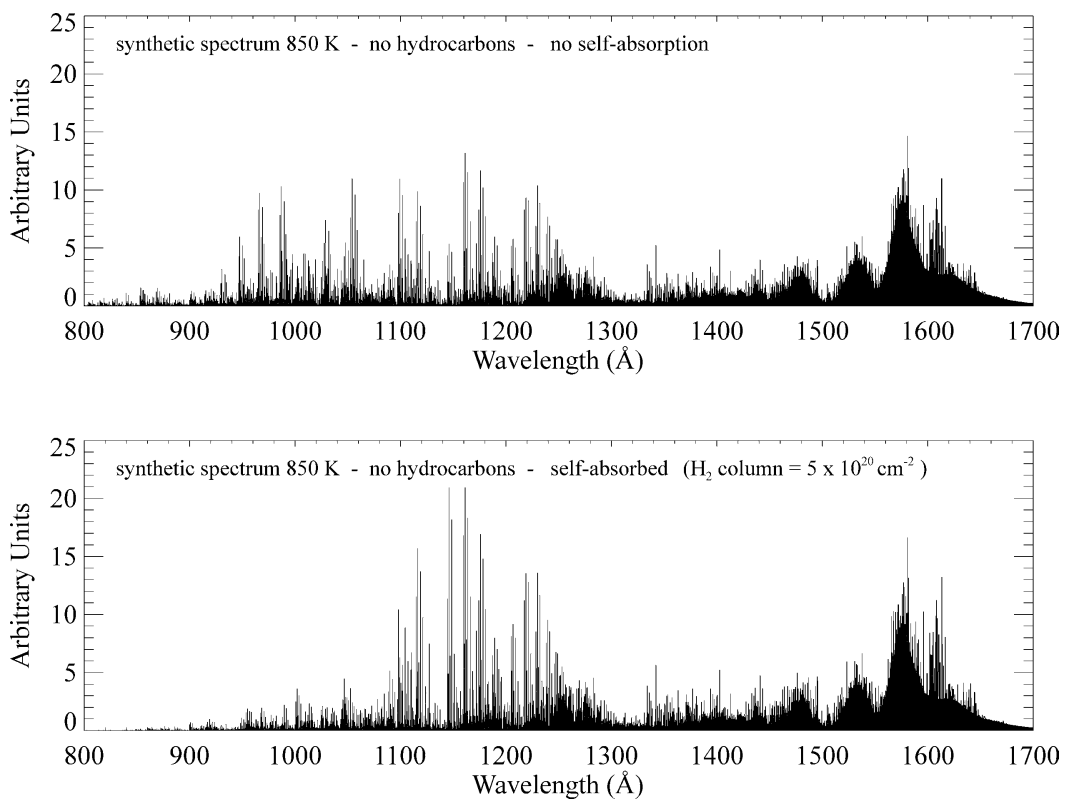

Fig. 6. Overview of the FUV + EUV synthetic spectrum, with and without self-absorption. Strong absorption is seen below $1100 \AA$ A. The total intensity of the spectrum is conserved and the energy absorbed at short wavelength is redistributed in the longer wavelength part of the spectrum.

Table 1

Main quantitative results for the single-layer models

\begin{tabular}{llll}
\hline Spectrum & $\begin{array}{l}\mathrm{H}_{2} \text { column (vertical value) } \\
\left(\mathrm{cm}^{-2}\right)\end{array}$ & $\begin{array}{l}\text { Corresponding pressure } \\
(\mu \mathrm{bar})\end{array}$ \\
\hline SiC2a $(920-970 \AA)$ & $3.2 \times 10^{17}<\mathbf{1 . 3} \times \mathbf{1 0}{ }^{18}<3.2 \times 10^{18}$ & $0.003<\mathbf{0 . 0 1 < 0 . 0 3}$ \\
LiF1a $(1030-1080 \AA)$ & $8.5 \times 10^{19}<\mathbf{1 . 5} \times \mathbf{1 0}^{20}<2.1 \times 10^{20}$ & $0.68<\mathbf{1 . 2 0}<1.67$ & $\begin{array}{l}\mathrm{H}_{2} \text { temperature } \\
\left(\mathrm{K}^{2}\right)\end{array}$ \\
LiF2a $(1090-1180 \AA)$ & $7.5 \times 10^{19}<\mathbf{1 . 3} \times \mathbf{1 0}^{20}<2.1 \times 10^{20}$ & $0.59<\mathbf{1 . 0 4}<1.67$ & - \\
\hline
\end{tabular}

To estimate the error in the parameters, we used the procedure described in Trafton et al. (1994). For the $\mathrm{H}_{2}$ column for example, we determine a confidence interval assuming that only the $\mathrm{H}_{2}$ column is uncertain while the temperature is fixed to its $\chi^{2}$ minimum value ( $\chi_{\text {min }}^{2}$ ). The difference between the $\chi^{2}$ obtained when we vary the $\mathrm{H}_{2}$ column and $\chi_{\min }^{2}$ follows a $\chi^{2}$ law with 1 degree of freedom. This law allows a determination of a $90 \%$ confidence interval, using the tabulated statistical tables. The same process is used to determine a $90 \%$ confidence interval for the temperature. 
connecting to $v^{\prime \prime}=0$. In order to avoid strong features from non- $\mathrm{H}_{2}$ lines, the spectral region longward of H Lyman $\gamma$ at $972.55 \AA$ was ignored. As seen in Fig. 2, the spectra obtained with the $\mathrm{SiC}$ segments are significantly noisier than the spectra obtained with the LiF segments. Through the analysis of the $920-970 \AA$ window, it was found that this spectral region was unreliable because of its low $\mathrm{S} / \mathrm{N}$ ratio. It did not allow to obtain reliable values of the $\mathrm{H}_{2}$ column and Tem, based on comparison between the data and model spectra. Thus, only the effects of self-absorption and temperature are described here, based on synthetic spectra. For sake of completeness, the $\mathrm{H}_{2}$ column and Tem, obtained on the base of the best fit, are mentioned but they will not be further discussed.

Figure 7 shows the comparison between the observed spectrum and the best fitting single layer synthetic spectrum. In addition to the $\mathrm{H}_{2}$ lines, features from the Earth dayglow and the H Lyman series are present in the observed spectrum. Application of the method described above gives rise to a best fit spectrum characterized by an emitting layer at $850 \mathrm{~K}$, with a slant $\mathrm{H}_{2}$ column of $6 \times 10^{18} \mathrm{~cm}^{-2}$. The main lines of the synthetic spectrum used to fit the data are identified in Fig. 8. This figure also compares the synthetic spectrum that gives the best fit and a synthetic spectrum at the same temperature with no self-absorption. It is seen that almost all the transitions in the spectral window are attenuated. Although lines connecting to $v^{\prime \prime}=0$ suffer from the strongest absorption, there is significant absorption for transitions connecting to $v^{\prime \prime}=1$ between 920 and $928 \AA$. At $900 \mathrm{~K}$, the synthetic spectral model predicts that for an unabsorbed spectrum, $56 \%$ of the total intensity in the 920 $970 \AA$ window is emitted in lines connecting to $v^{\prime \prime}=0$ and $21 \%$ of the intensity lies in lines connecting to $v^{\prime \prime}=1$. As shown in Fig. 9, for an $\mathrm{H}_{2}$ column of $1 \times 10^{19} \mathrm{~cm}^{-2}$, this fraction drops to $0.2 \%$ for $v^{\prime \prime}=0$ and $18.8 \%$ for $v^{\prime \prime}=1$, and reaches only $10^{-4} \%$ for $v^{\prime \prime}=0$ and $0.03 \%$ for $v^{\prime \prime}=1$ when the $\mathrm{H}_{2}$ column is $1 \times 10^{23} \mathrm{~cm}^{-2}$. It is also seen that for an $\mathrm{H}_{2}$ column of $10^{19} \mathrm{~cm}^{-2}$, the total intensity of the $v^{\prime \prime}=0$ lines represents only about $0.2 \%$ of the intensity of the unabsorbed spectrum. If ones defines a group of lines as saturated when the intensity of this group drops to $\sim 1 \%$ of the unabsorbed intensity, we can consider the lines connecting to $v^{\prime \prime}=0$ as saturated for an $\mathrm{H}_{2}$ column higher than $1 \times 10^{19} \mathrm{~cm}^{-2}$. Attenuation of the $v^{\prime \prime}=1$ lines mainly occurs in the 920-928 $\AA$ window, which is the noisiest region of the whole spectral window. Figure 9 also shows that at $900 \mathrm{~K}$ (compared to $400 \mathrm{~K}$ ), the attenuation increases faster for lines connecting to $v^{\prime \prime}=1$. This is consistent with the fact that the population of high vibrational levels increases with temperature.

\subsubsection{0-1080 ̊ window-LiFla}

For this spectral window, the LiF1a segment was selected since it has the highest signal to noise ratio. We also ignore the spectrum below $1030 \AA$ to avoid the bright HLy $\beta$ line at $1025.73 \AA$. As for the $920-970 \AA$ region, the spectrum is contaminated by Earth dayglow lines, as well as features from the jovian dayglow and the Io torus. The best fit, presented in Fig. 10, is obtained with a Tem of $800 \mathrm{~K}$ and an $\mathrm{H}_{2}$ slant column of $7 \times 10^{20} \mathrm{~cm}^{-2}$. As for the previous spectral window, the small variation of the methane cross-section does not allow an accurate determination of the $\mathrm{CH}_{4}$ column. Figure 11 compares the best fit model with a model at the same Tem, not self-absorbed. The main discrepancies between the observed spectrum and the fit are explained by the jovian and Earth dayglow and Io torus contamination, especially at 1032, 1062.6, 1071.5, and $1077 \AA$. Some lines, such as the $W(0,1) R(1)$ and $L(5,0) P(5)$ at $1052.6 \AA$ and the $L(4,0) P(3)$ at $1056.3 \AA$, suggest that the model is not self-absorbed enough, while other features suggest a correct attenuation by self-absorption (the $L(5,0) P(3)$ at $1043.5 \AA$
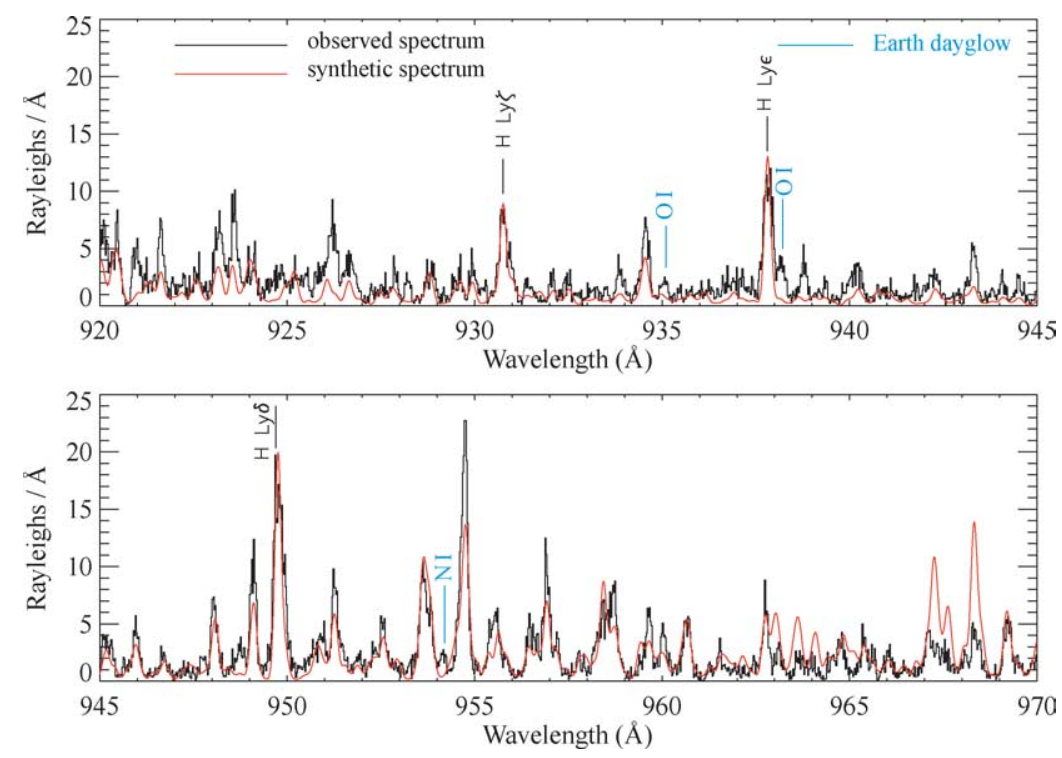

Fig. 7. Spectrum obtained with the FUSE SiC2a segment overlaid by the best fit model: Tem $=850 \mathrm{~K}, \mathrm{H}_{2}$ column $=6 \times 10^{18} \mathrm{~cm}^{-2}$. Note that the observed spectrum is very noisy. 

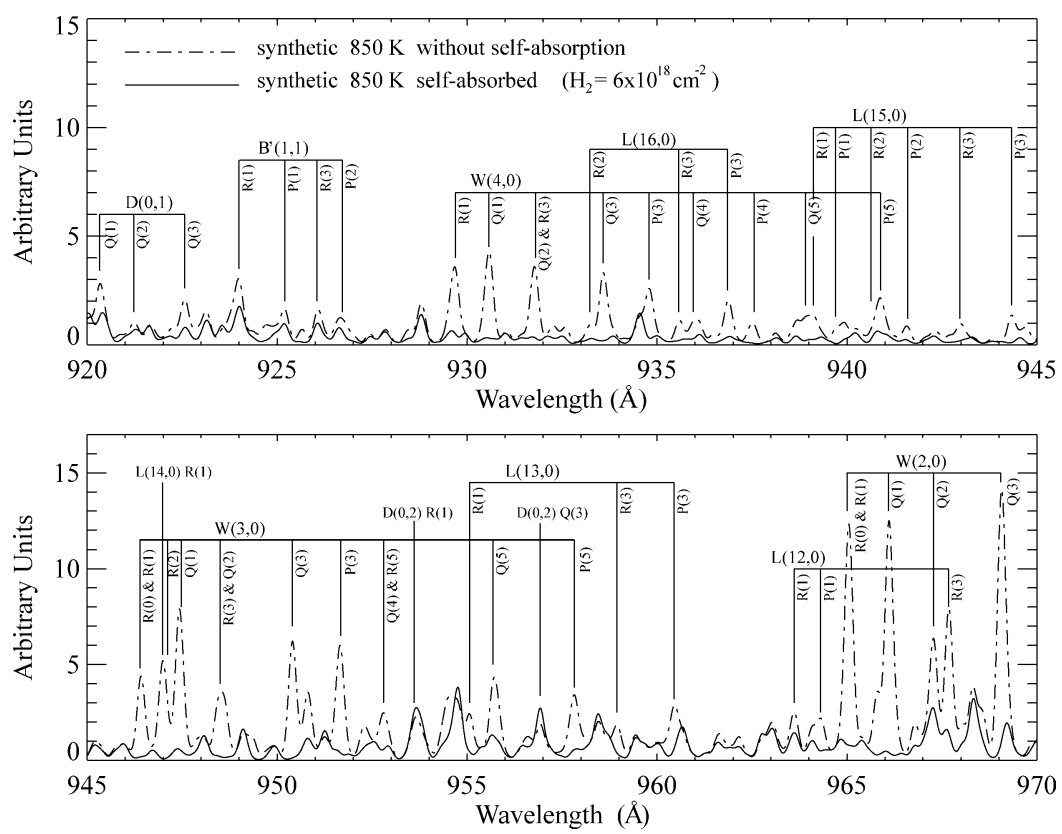

Fig. 8. Comparative plots of two single-layer models in the spectral window of the SiC2a segment. The best fit model is compared to a model obtained with the same parameters but not self-absorbed. It is seen that most of the lines, which are the result of transitions connecting to $v^{\prime \prime}=0$ and $v^{\prime \prime}=1$, are strongly absorbed.

or the $W(0,1) P(5)$ at $1065 \AA$ for example). This apparent contradiction indicates that the best fit we obtain is a compromise between regions of the spectrum which behave oppositely (some line intensities are improving while others are getting worse) when the parameters of the model are varied. Figure 12 shows that at $900 \mathrm{~K}$, lines connecting to $v^{\prime \prime}=0$ are saturated near $1 \times 10^{18} \mathrm{~cm}^{-2}$ (meaning that the total intensity of the lines connecting to $v^{\prime \prime}=0$ in the spectral window is about $1 \%$ of the non-self-absorbed intensity). The lines connecting to $v^{\prime \prime}=1$ are saturated between $1 \times 10^{20}$ and $1 \times 10^{21} \mathrm{~cm}^{-2}$, which implies that in the range of parameters that gives the best fit spectrum, $\chi^{2}$ is mainly sensitive to lines connecting to $v^{\prime \prime}=2$ (as clearly seen in Fig. 12c), which represent between 20 and $30 \%$ of the total intensity in the spectral window.

\subsubsection{0-1180 ̊ window-LiF2a}

The last spectral window we analyzed is the entire LiF2a segment, from 1090 to $1180 \AA$. Though the signal to noise ratio of the LiF2a and $\mathrm{LiF} 1 \mathrm{~b}$ segments is comparable, the $\mathrm{LiF} 1 \mathrm{~b}$ channel is more susceptible to calibration uncertainty for non-point sources and was not used. Some differential wavelength shifts were found in this spectrum and were corrected by comparing the observed lines with a synthetic spectrum. Using the method described below, the fit minimizing $\chi^{2}$ is shown in Fig. 13. In this spectral region, the variation of the $\mathrm{CH}_{4}$ absorption cross-section with wavelength allows one to derive a $\mathrm{CH}_{4}$ column. We find a $\mathrm{CH}_{4}$ slant column of $1 \times 10^{16} \mathrm{~cm}^{-2}$ and an $\mathrm{H}_{2}$ slant column of $6.2 \times 10^{20}$ for a Tem of $800 \mathrm{~K}$. It corresponds to a vertical column of $2.1 \times 10^{15} \mathrm{~cm}^{-2}$ and $1.3 \times 10^{20} \mathrm{~cm}^{-2}$ for $\mathrm{CH}_{4}$ and $\mathrm{H}_{2}$, respectively. Applying the hydrostatic law, it is found that the $\mathrm{H}_{2}$ column corresponds to a pressure level of $\sim 1 \mu \mathrm{bar}$. To find the pressure level corresponding to the $\mathrm{CH}_{4}$ column found here, we need to use an atmospheric model. Using the modified NEB model by Gladstone et al. (1996), it is found that a $\mathrm{CH}_{4}$ column of $2.1 \times 10^{15} \mathrm{~cm}^{-2}$ leads to a pressure of $\sim 2 \mu \mathrm{bar}$, close to the pressure level calculated from the $\mathrm{H}_{2}$ column. These two numbers are comparable and show that the parameters derived from the single-layer model are consistent, although it should be noted that the single-layer model does not reflect the vertical extend of the atmosphere, while photons are likely produced and absorbed at different altitudes. The correspondence between the methane and the $\mathrm{H}_{2}$ columns and their interpretation is therefore biased in the single-layer approximation. The only way to derive a relationship between the $\mathrm{CH}_{4}$ and $\mathrm{H}_{2}$ columns and the emission peak level would be to use a spectrum that contains both the short and long-wavelength FUV spectral regions in a 1-D atmosphere. This point will be discussed in the next section with the analysis of the HUT auroral spectrum.

The fit so obtained is excellent and the main discrepancies can be explained by the jovian dayglow lines present in the spectrum. The discrepant features at 1092.7 and $1167 \AA$ are not explained. Although the $W(0,2)$ lines from 1100 to $1110 \AA$ are well reproduced, the $W(0,2) R(1)$ and $W(0,2) Q(1)$ lines at 1098 and $1099.5 \AA$ present the main discrepancies in the spectrum. They may be explained by departure from the thermodynamic equilibrium (LTE) distribution of the $\mathrm{H}_{2}$ molecules in the $v^{\prime \prime}=2$ level or by other factors, as suggested by Wolven and Feldman (1998).

At a temperature of $900 \mathrm{~K}$, the lines connecting to $v^{\prime \prime}=0$ are quickly saturated for columns higher than $1 \times 10^{19} \mathrm{~cm}^{-2}$, 


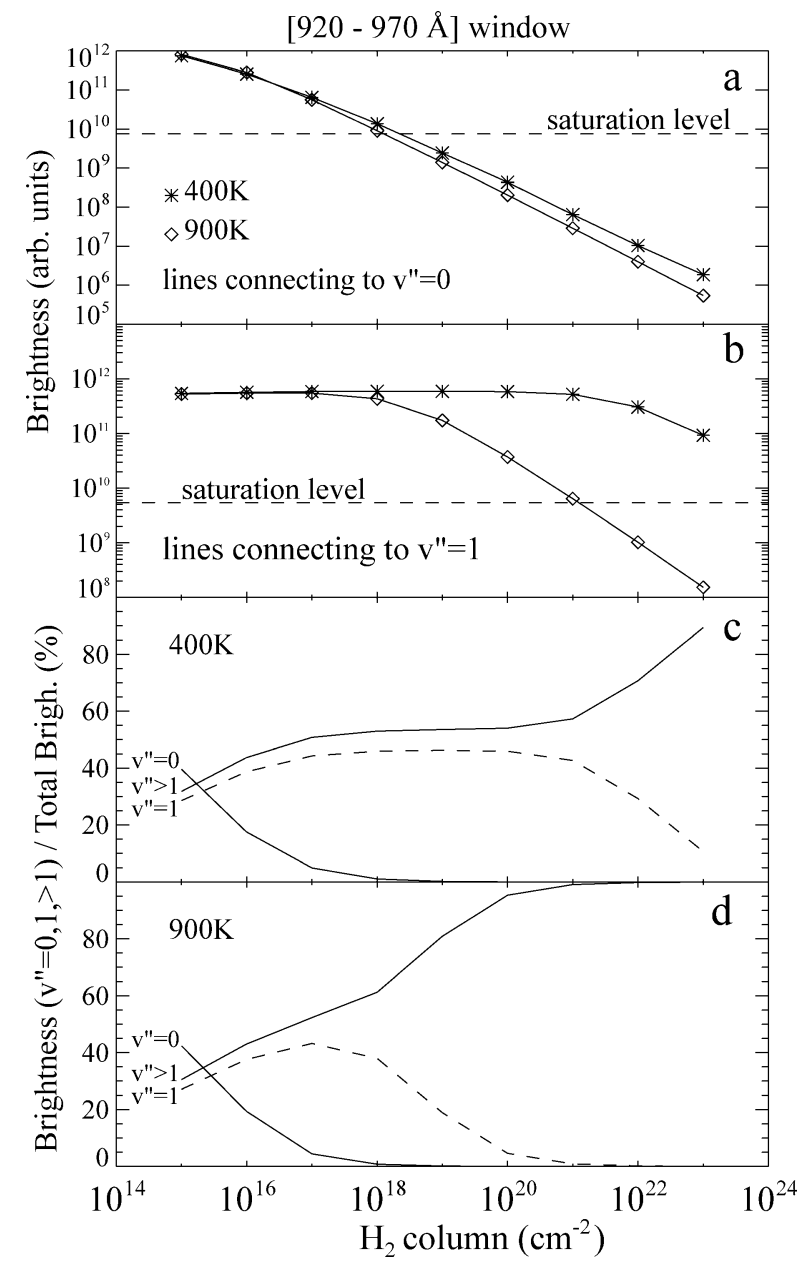

Fig. 9. Influence of the $\mathrm{H}_{2}$ column and the temperature on self-absorption in the 920-9970 $\AA$ window. For better legibility, the brightness is not shown when the $\mathrm{H}_{2}$ column $=0$. (a) Absolute intensity variation of all the lines connecting to the $v^{\prime \prime}=0 \mathrm{H}_{2}$ ground level in the spectral window, using simulations with synthetic spectra. The lines are quickly saturated near $10^{18} \mathrm{~cm}^{-2}$, for both temperatures, though it is felt that the lines are still strongly absorbed for columns higher that $10^{18} \mathrm{~cm}^{-2}$, because of the Y-axis $\log$ scale. (b) Same as (a) for lines connecting to $v^{\prime \prime}=1$. The effect of temperature is more obvious than for the $v^{\prime \prime}=0$ lines. At $400 \mathrm{~K}$, the absorption of the $v^{\prime \prime}=1$ lines is weak compared to the absorption at $900 \mathrm{~K}$, where the $v^{\prime \prime}=1$ lines reach the saturation level near $10^{21} \mathrm{~cm}^{-2}$. (c) Variation, at $400 \mathrm{~K}$, of the contribution of the lines connecting to $v^{\prime \prime}=0,1$ and $>1$ to the total intensity of the 920-970 $\AA$ spectral window. (d) Same as (c) for a temperature of $900 \mathrm{~K}$. Near the $\mathrm{H}_{2}$ column that provides the SiC2a best fit $\left(6 \times 10^{18} \mathrm{~cm}^{-2}\right)$, the intensity variation of the $v^{\prime \prime}=1$ lines is maximum thus the $v^{\prime \prime}=1$ lines drive the $\chi^{2}$ behavior. It should be noted that the increase of the contribution of the lines connecting to vibrational levels higher than 1 is due to the decrease of the contribution of the $v^{\prime \prime}=1$ lines. There is very little absolute variation of the lines connecting to $v^{\prime \prime}>1$ in this spectral window.

while the lines connecting to $v^{\prime \prime}=1$ saturate between $1 \times$ $10^{20}$ and $1 \times 10^{21} \mathrm{~cm}^{-2}$. Figure 14 shows the best fit model along with an unabsorbed model. Lines at 1090.5, 1104.7, and $1114 \AA$ for example indicate that self-absorption of the $v^{\prime \prime}=1$ lines is well reproduced. Lines connecting to $v^{\prime \prime}=2$ are not saturated at the $\mathrm{H}_{2}$ column providing the best fit (the intensity of all the $v^{\prime \prime}=2$ lines at $1 \times 10^{21} \mathrm{~cm}^{-2}$ is $64 \%$ of the non-self-absorbed intensity), but their contribution to the total intensity in the spectral window is only $9 \%$. The $\chi^{2}$ near the best fit parameters is apparently more influenced by lines connecting to $v^{\prime \prime}=3$, which represent $36 \%$ of the total intensity at $900 \mathrm{~K}$ and $1 \times 10^{21} \mathrm{~cm}^{-2}$ of $\mathrm{H}_{2}$ (Fig. 15). Figures 14 and 15 show that the $v^{\prime \prime}=3$ transitions are not absorbed, but brighten, for all temperatures and $\mathrm{H}_{2}$ column values found in the jovian auroral atmosphere owing to the fact that an important part of the absorbed emission is redistributed to transitions connecting to $v^{\prime \prime} \geqslant 3$.

\subsubsection{Sensitivity to the temperature of $\mathrm{H}_{2}$ self-absorption}

The approach described above assumes that the temperature controlling the population of the absorbing molecules $(T s a)$ is the same as the temperature of the emission (Tem). Tests were made to uncouple the temperature of the emission layer and the temperature of the $\mathrm{H}_{2}$ absorbing region. Nested loops were used with Tem, $\mathrm{H}_{2}$ column and Tsa as free variable parameters. For the $\mathrm{SiC} 2 \mathrm{a}$ spectrum, a best fit is obtained for a Tem of $900 \mathrm{~K}$, an $\mathrm{H}_{2}$ column of $1 \times 10^{20} \mathrm{~cm}^{-2}$ and $T s a$ of $600 \mathrm{~K}$. A comparison between this best fit and the best fit obtained in Section 4.1.1 shows that the two models are almost identical. This result has no physical meaning since the temperature of the atmosphere rises monotonically with the altitude in the thermosphere, up to the exobase. Consequently, any value Tem $>T s a$ is unrealistic, since selfabsorption can only occur above or close to the altitude of emission. It confirms the unreliability of the 920-970 A region. The slightly smaller $\chi^{2}$ obtained here is more likely due to the addition of a degree of freedom in the fitting procedure than to a significant improvement of the model. For information, when Tsa is varied with Tem and $\mathrm{H}_{2}$ column fixed to their best fit values $\left(850 \mathrm{~K}\right.$ and $6 \times 10^{18} \mathrm{~cm}^{-2}$, respectively), the model minimizing $\chi^{2}$ is obtained for a $T s a$ of $850 \mathrm{~K}$.

The same method was applied to the LiF1a spectrum. A best fit is obtained for a Tem of $750 \mathrm{~K}$, an $\mathrm{H}_{2}$ column of $5 \times 10^{19} \mathrm{~cm}^{-2}$ and a Tsa of $1000 \mathrm{~K}$. Again, a comparison between this best fit model and the best model previously obtained in Section 4.1.2 with Tsa fixed to the value of Tem shows very little difference between the two models. As for the previous case, the improvement of the fit is a consequence of the addition of a new free parameter rather than a real improvement of the model. When the Tem and $\mathrm{H}_{2}$ column are fixed to their best fit values $(800 \mathrm{~K}$ and $7 \times 10^{20} \mathrm{~cm}^{-2}$, respectively), Tsa minimizing $\chi^{2}$ is $800 \mathrm{~K}$.

When applied to the LiF2a spectrum, this method shows that a best fit is obtained with the same parameter values as the values obtained with Tsa fixed to the value of Tem $(800 \mathrm{~K})$.

\subsubsection{Summary of the single-layer study}

(1) The $\chi^{2}$ that determines the best fit parameters (Tem, $\mathrm{H}_{2}$ column and $\mathrm{CH}_{4}$ column) is mainly dominated by lines connecting to $v^{\prime \prime}=1$ in the 920-970 $\AA$ window, $v^{\prime \prime}=2$ in the 1030-1080 ̊ window, $v^{\prime \prime}=2$ and $v^{\prime \prime}=3$ in the 1090- 

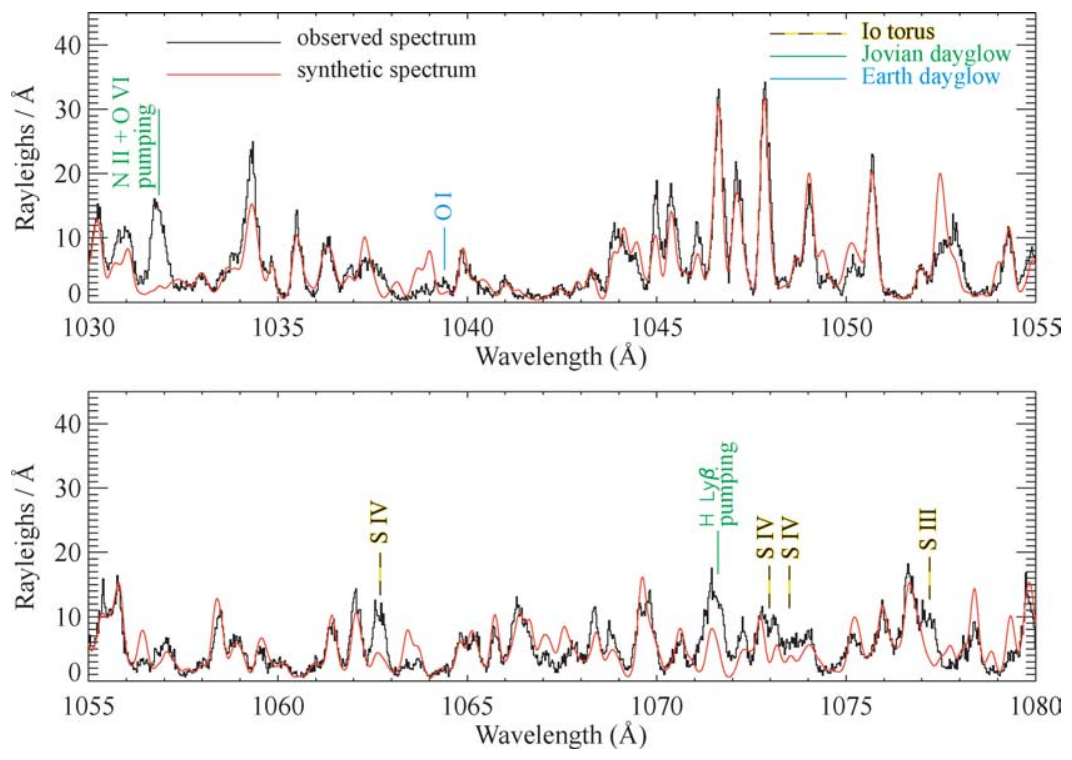

Fig. 10. LiF1a spectrum and best fit model: Tem $=800 \mathrm{~K}, \mathrm{H}_{2}$ column $=7 \times 10^{20} \mathrm{~cm}^{-2}$.
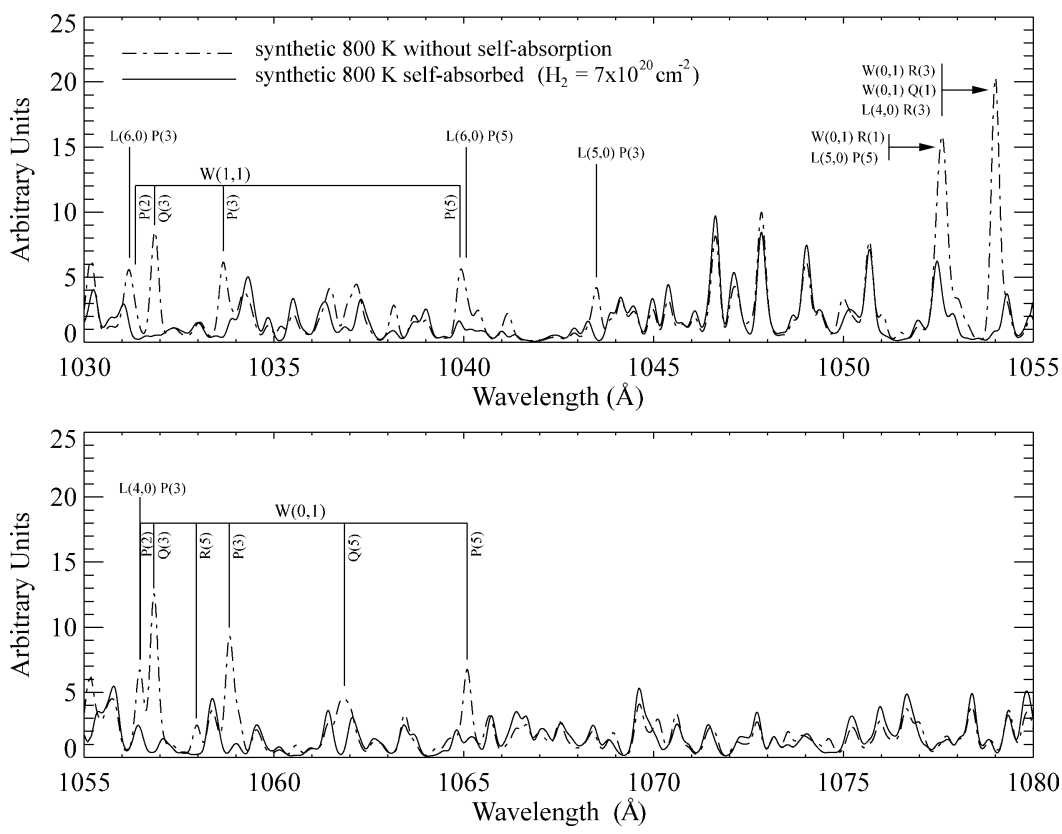

Fig. 11. Best fit model of the LiF1a spectrum is compared to a model at the same temperature and not self-absorbed. Few lines connecting to $v^{\prime \prime}=0$ and $v^{\prime \prime}=1$ are strongly absorbed. Only the main absorbed transitions are identified.

$1180 \AA$ A window. For a given vibrational lower level $v^{\prime \prime}, \chi^{2}$ is sensitive to the combination of rapid intensity variations with $\mathrm{H}_{2}$ column and a significant proportion of lines connecting to $v^{\prime \prime}$ in the spectral window.

(2) "Line-by line" interpretation is difficult because most of the lines in the spectra are blended with different transitions.

(3) Figures 9,12 , and 15 clearly show that the redistribution of $\mathrm{H}_{2}$ photons from higher transitions to lower transitions depends on the $\mathrm{H}_{2}$ column and $T s a$. For example, at $400 \mathrm{~K}$, lines connecting to $v^{\prime \prime}=2$ in the $1090-1180 \AA$ spectrum become more intense with increasing $\mathrm{H}_{2}$ column while at $900 \mathrm{~K}$, these lines are strongly absorbed when the $\mathrm{H}_{2}$ column exceeds $1 \times 10^{19} \mathrm{~cm}^{-2}$ (Fig. 12c).

(4) The main effect of the temperature is to modify the rovibrational population in the $\mathrm{H}_{2}$ electronic ground state. A temperature increase populates higher vibrational levels (mainly $v^{\prime \prime}=1,2$, and 3) and makes transitions connecting to these levels more sensitive to self-absorption, both in absorption and fluorescence.

(5) Tests were made to uncouple the temperature of the emitting molecules (Tem) and the temperature controlling the population of the absorbing molecules ( $T_{s} a$ ). They show that including $T s a$ as a free parameter in the fitting procedure 


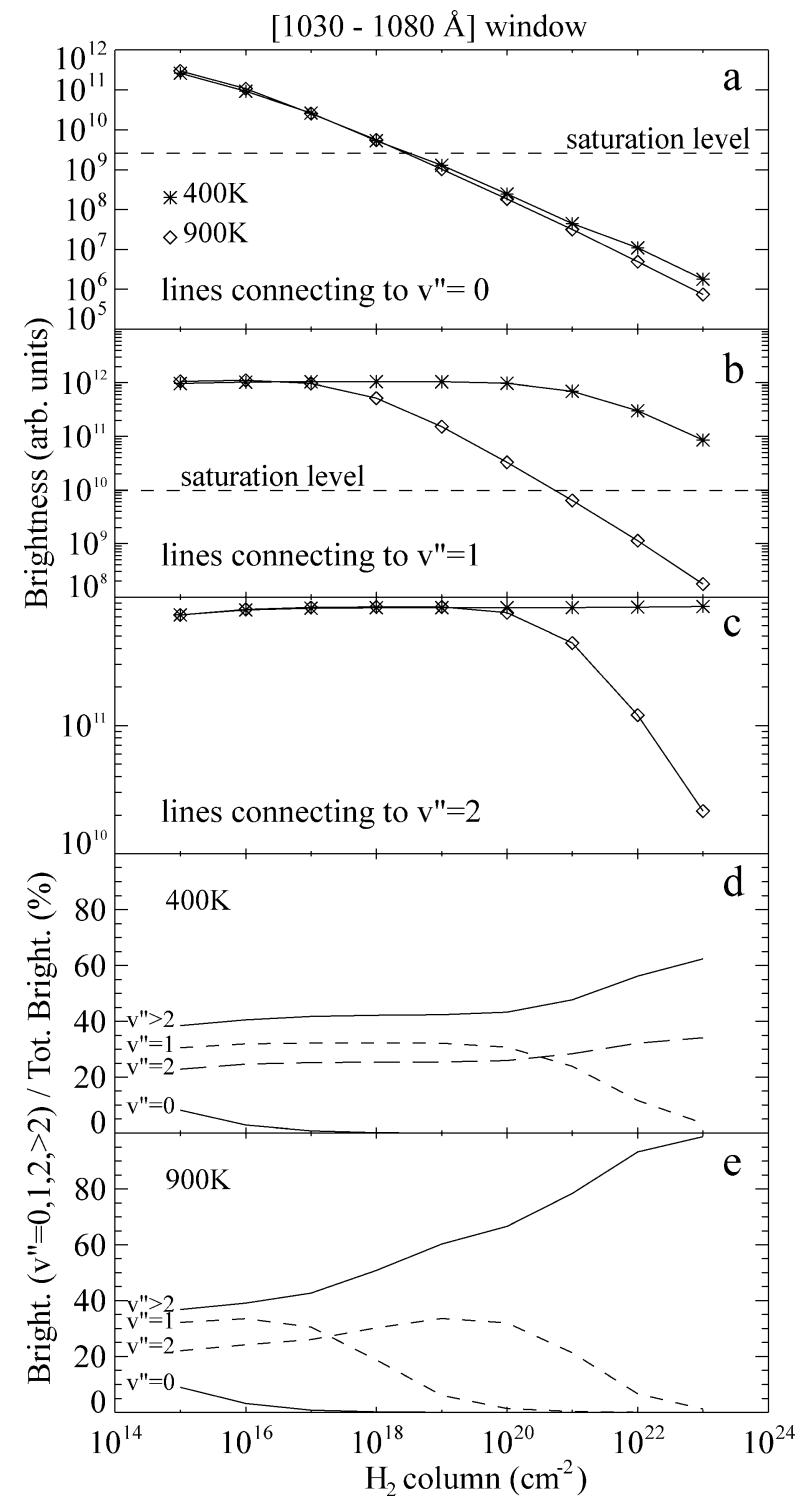

Fig. 12. Influence of the $\mathrm{H}_{2}$ column and the temperature on self-absorption in the 1030-1080 $\AA$ window. (a) Same as Fig. 9a. The saturation level is reached for an $\mathrm{H}_{2}$ column close to $10^{18} \mathrm{~cm}^{-2}$. (b) Same as Fig. 9b. The behavior of the lines connecting to $v^{\prime \prime}=1$ is comparable to the behavior of these lines in the 920-970 $\AA$ window. (c) Absolute variation of lines connecting to $v^{\prime \prime}=2$. The effect of temperature is clearly seen. When the $\mathrm{H}_{2}$ temperature is in the order of $400 \mathrm{~K}$, the gas has very little effects on self-absorption. At $900 \mathrm{~K}$, the population of the $\mathrm{H}_{2}$ molecules in the $v^{\prime \prime}=2$ vibrational level increases and it provides a strong absorption of the photons connecting to this vibrational level. (d) Same as Fig. 9c in the 1030-1080 spectral window. (e) The best fit spectrum of the LiF2a is obtained for an $\mathrm{H}_{2}$ column of $6.2 \times 10^{20} \mathrm{~cm}^{-2}$. For this value of the $\mathrm{H}_{2}$ column, the lines connecting to $v^{\prime \prime}=0$ and $v^{\prime \prime}=1$ are saturated and the intensity variations are due to lines connecting to $v^{\prime \prime}=2$. As for Fig. 9d, the contribution of the lines connecting to levels higher than $v^{\prime \prime}=2$ in the spectral window is due to the decrease of the contribution of the $v^{\prime \prime}=2$ lines rather than a significant variation of the lines connecting to $v^{\prime \prime}>2$.

does not lead to significant improvement. The similarities between models with $T s a$ fixed or freely varying indicate that a given value of the transmission coefficient can be obtained with different sets of values of the $\left(\mathrm{H}_{2}\right.$ column-Tsa) pair.
This is a numerical effect and it does not bring any further understanding of the jovian atmosphere. We note that with $T s a$ as a free parameter, the best fit spectra are obtained with the same value of Tem (within $50 \mathrm{~K}$ ), as in the case where Tsa is fixed. Also, when Tem and $\mathrm{H}_{2}$ column are fixed to their best fit value listed in Sections 4.1.1-4.1.3, the value of Tsa (as free parameter) that minimize $\chi^{2}$ is the same as Tem. These results suggest that emission and absorption of photons occur approximately at the same altitude, as expected from the rapid exponential drop of $\mathrm{H}_{2}$ density with altitude. As a consequence, we find no need to use a Tsa value that is twice the gas temperature (Tem), as was done in Ajello et al. (2001).

(6) As short-wavelength FUV auroral emission is strongly absorbed by methane, the $\mathrm{H}_{2}$ column derived from selfabsorbed short-wavelength FUV spectra is located above the $\mathrm{CH}_{4}$ homopause. Since $\mathrm{H}_{2}$ is the major constituent in the jovian atmosphere, the $\mathrm{H}_{2}$ columns found from the LiF1b and LiF2a spectra $\left(\sim 1 \times 10^{20} \mathrm{~cm}^{-2}\right)$ can be directly converted in pressure values. It is found that an $\mathrm{H}_{2}$ column of $1 \times 10^{20} \mathrm{~cm}^{-2}$ corresponds to $\sim 1 \mu \mathrm{bar}$. When compared to the modified NEB model (Gladstone et al., 1996), these values corresponds to an altitude close to the methane homopause, which is located near $5 \mu$ bar in the modified NEB model. The $\mathrm{H}_{2}$ vertical column found for the $\mathrm{SiC} 2 \mathrm{a}$ spectrum $\left(1.3 \times 10^{18} \mathrm{~cm}^{-2}\right)$ corresponds to an altitude of $\sim 0.01 \mu$ bar. This suggests that the auroral emission in this spectral window is far above the methane homopause, but as mentioned before, the low signal to noise ratio of the $\mathrm{SiC} 2 \mathrm{a}$ spectrum does not allow a definite determination of the $\mathrm{H}_{2}$ column. A fit of the $\mathrm{SiC} 2 \mathrm{a}$ spectrum was made with the parameters that best fit LiF1a segment $\left(T=800 \mathrm{~K}\right.$ and $\mathrm{H}_{2}$ column $=7 \times 10^{20} \mathrm{~cm}^{-2}$ ). The difference by eye between the two fits in the first half of the spectral window (which is the noisiest part) is very small and only the $\chi^{2}$ allows a choice between the two models.

\subsection{Multi-layer analysis}

The multi-layer approach is more realistic since it directly combines the synthetic spectral generator and the 1D energy degradation model described by Grodent et al. (2001, private communication, 2001) adapted from the NEB model by Gladstone et al. (1996). This 1-D model considers an electron energy flux at the top of the atmosphere and self-consistently calculates the vertical temperature profile, the composition structure, and the degraded energy flux of the electrons as they penetrate deeper into the jovian atmosphere. In this scheme, the main input parameter is the energy spectrum of the precipitating electrons. Among other results, the 1-D model calculates the local electron energy distribution, the $\mathrm{H}_{2}, \mathrm{CH}_{4}$, and $\mathrm{C}_{2} \mathrm{H}_{2}$ density profiles, and the gas temperature at 200 pressure/altitude levels. These outputs are rebinned to fit in the 20 atmospheric layers considered in the spectral generator. The resulting spectrum, which consists of the sum of the 20 spectra weighted by the elec- 

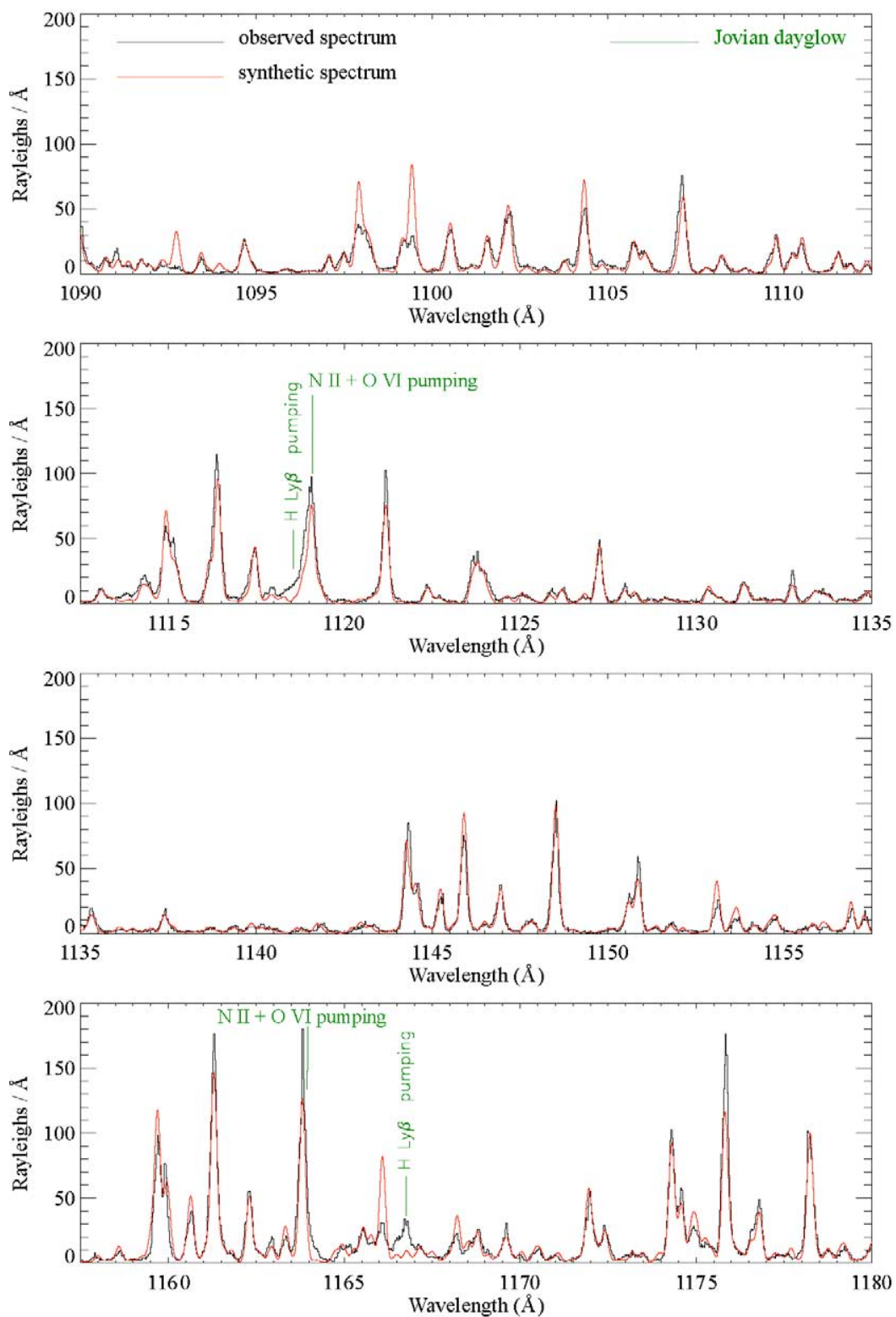

Fig. 13. Comparison between the LiF2a spectrum and best fit model: Tem $=800 \mathrm{~K}, \mathrm{H}_{2}$ column $=6.2 \times 10^{20} \mathrm{~cm}^{-2}$.

tron energy flux, is then compared to the observed data (Dols et al., 2000; Gustin et al., 2002). In this approach, Tsa and Tem were fixed to the $\mathrm{H}_{2}$ temperature provided by the 1-D model. Several energy distributions were tested in order to fit the three FUSE spectral windows described above. The main observational constraints imposed on the energy distribution were to obtain a 1-D atmosphere providing sufficiently self-absorbed synthetic spectra. In this scheme, simultaneous high values for the $\mathrm{H}_{2}$ column and temperature near the homopause were required, along with a reasonable value of the color ratio (see Fig. 2 in Gérard et al., 2002). These constraints allow us to considerably restrict the specifications of the primary precipitated electrons, in terms of energy flux $(\Phi)$ and characteristic energy $\left(E_{0}\right)$.

The best fit spectra obtained for the three windows are very close to the best synthetic spectra obtained with the one- layer analysis and therefore are not shown. The similarity of the one-layer and the multi-layer model spectra indicates that the best fits reached by the multi-layer approach give a good estimate of the energy spectrum of the precipitating electrons. An important point is that the scaling factors applied to the synthetic spectrum to fit the data are identical within $2 \%$ for each spectral window. This implies that fitting the overall FUSE spectral window would lead to the same result. It also implies that the temperature, the $\mathrm{CH}_{4}$ column and the $\mathrm{H}_{2}$ column associated with the three studied spectral windows reach a consistent value and provide an overall good fit to the data.

Figure 16 shows the characteristics of the model atmosphere used in this study. The initial electron energy distribution is given in Table 2. It combines a series of six Maxwellian distributions. The addition of several Maxwel- 

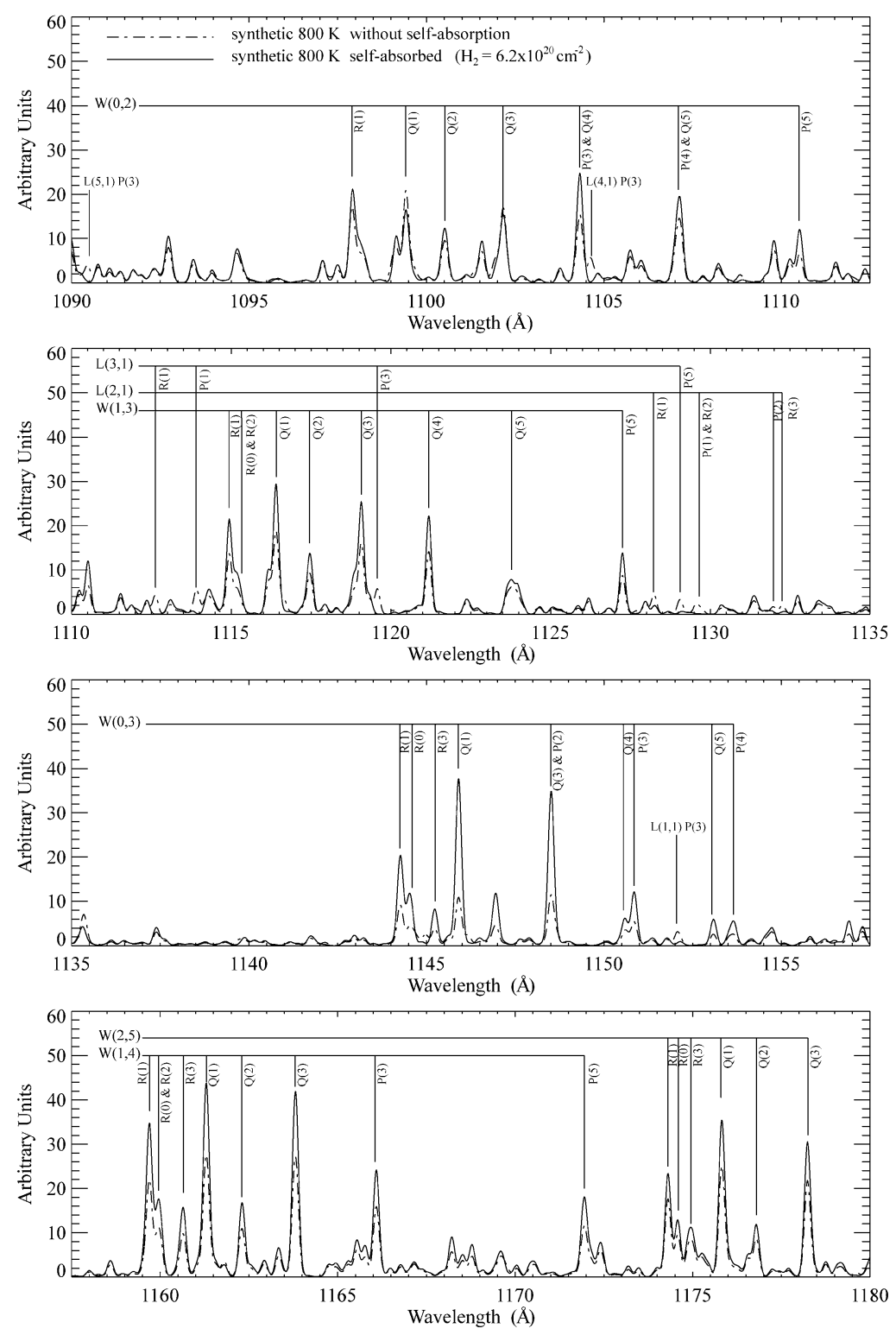

Fig. 14. The single-layer model that best fit the LiF2a spectrum is compared to a single-layer model at the same temperature and not self-absorbed. While the lines connecting to $v^{\prime \prime}=2$ can be both absorbed or more intense by a fluorescence process, the lines connecting to $v^{\prime \prime} \geqslant 3$ are also influenced by self-absorption. They are brighter and get the energy from self-absorbed lines coming from the shorter wavelength part of the spectrum.

lian components allowed to easily modify the shape of the energy spectrum of the primary electrons. Since the $\mathrm{H}_{2}$ density and temperature profiles of the atmosphere (and thus the resulting synthetic spectrum) are very sensitive to this input, our best electron energy distribution was obtained after some trial shots, until a good fit was achieved. An energy distribution composed of multiple Maxwellians is always somewhat arbitrary, but it provides a suitable, though not unique, description of the primary electron's energy spectrum. Each Maxwellian used here has a physical meaning that can be interpreted as follows. The dominant Maxwellian component $\left(100 \mathrm{erg} \mathrm{cm}^{-2} \mathrm{~s}^{-1}\right)$ has a characteristic energy of $40 \mathrm{keV}$ and determines the altitude of the UV emission peak. The components at 1,4 , and $9 \mathrm{keV}$ contribute to the emission appearing above the $10 \mu \mathrm{bar}$ level and heat up the upper at- mosphere. The $25 \mathrm{keV}$ component is included to enhance the temperature gradient and emphasize the $\mathrm{H}_{2}$ emission rate just above the methane homopause. The $100 \mathrm{keV}$ component slightly changes the $\mathrm{H}_{2}$ emission profile below the altitude peak and refines the $\mathrm{H}_{2}$ profile in order to keep the color ratio in the range of previously observed values. Consequently, the low altitude emission mainly controls the color ratio (2.9 at $60^{\circ}$ zenith angle), while the high altitude emission near $500 \mathrm{~km}$ is necessary to provide, at the same time, a hot component, characterized by highly populated $v^{\prime \prime}=1$ and $v^{\prime \prime}=2$ vibrational levels of the $\mathrm{H}_{2}$ ground state, and a $\mathrm{H}_{2}$ column that is sufficiently large to account for the self-absorption of the FUSE spectra. It should be mentioned that although the total energy input flux is considerable $\left(278 \mathrm{erg} \mathrm{cm}^{-2} \mathrm{~s}^{-1}\right)$, it is in the range of the 45 to $450 \mathrm{erg} \mathrm{cm}^{-2} \mathrm{~s}^{-1}$ determined 


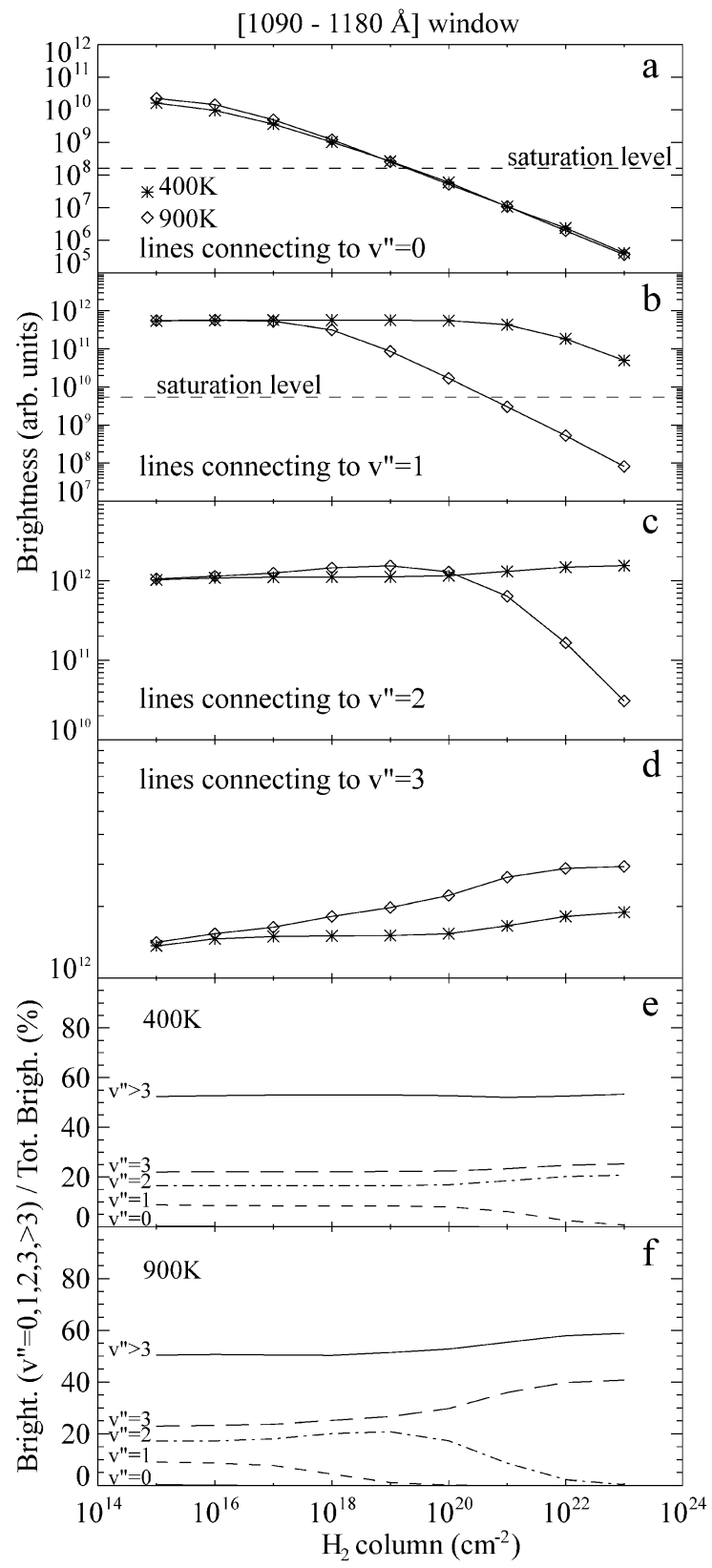

Fig. 15. Influence of the $\mathrm{H}_{2}$ column and the temperature on self-absorption in the 1090-1180 $\AA$ window. (a) Same as Figs. 9a and 12a. (b) Same as Figs. 9b and 12b. (c) Same as Figs. 9c and 12c. (d) Absolute variation of the lines connecting to $v^{\prime \prime}=3$. The effect of self-absorption is to increase the intensity of these lines (at temperatures and $\mathrm{H}_{2}$ columns found near the jovian homopause). That is, the lines that are self-absorbed in the shorter wavelength part of the UV spectrum are redistributed at longer wavelength. (e) The contribution of the lines connecting to all vibrational levels remain nearly constant up to an $\mathrm{H}_{2}$ column of $5 \times 10^{21} \mathrm{~cm}^{-2}$. (f) The effect of temperature is clearly seen. The absorption of lines connecting to $v^{\prime \prime}<3$ becomes effective near $10^{20} \mathrm{~cm}^{-2}$ and the lines connecting to $v^{\prime \prime}=3 \mathrm{dom}-$ inate in this spectral window. They contribute $\sim 35 \%$ of the intensity near the best fit values of temperature and $\mathrm{H}_{2}$ column.

by Clarke et al. (1996) from FUV images. Assuming that an electron flux of $1 \mathrm{erg} \mathrm{cm}^{-2} \mathrm{~s}^{-1}$ produces an auroral brightness of 10 kilo Rayleighs (kR) (Gérard and Singh, 1982; Waite et al., 1983), the total unabsorbed brightness of the aurora produced by the multi-layer model is $2.8 \mathrm{Mega}$ Rayleighs (MR). On the other hand, taking the LiF2a spectrum as a reference, the total intensity in the 1090-1180 spectra window is $\sim 1.1 \mathrm{kR}$. An estimate of the observed auroral brightness in the $800-1700$ spectral $\AA$ window can be obtained using the synthetic spectrum that best fits LiF2a. This synthetic spectrum shows that the intensity ratio between the 1090-1180 and the 800-1700 $\AA$ windows is 54.8 . The total brightness is thus $\sim 61 \mathrm{kR}$ for the $800-1700 \AA$ observed spectrum, attenuated by methane. Taking into account the ratio of 5 between the absorbed and unabsorbed $\mathrm{H}_{2}$ volume emission rate in the present case (see Fig. 16b), the total unabsorbed intensity of the FUV $\mathrm{H}_{2}$ emission is $\sim 305 \mathrm{kR}$. This brightness needs to be corrected for the filling factor, as the aurora does not completely fill the LWRS aperture. Considering that the auroral emission is defined by the pixels enclosed by the main oval, the mean ratio between the auroral area and the total LWRS area is $\sim 0.1$. The total auroral brightness derived from the observation is thus $\sim 3 \mathrm{MR}$. This estimate is close to the brightness of $\sim 2.8 \mathrm{MR}$ deduced from the flux of precipitated electrons. The value of the filling factor is the key point, as it cannot be determined from the FUSE observations. Though this filling factor is uncertain, a value of 10 is reasonable since the geometry of observation is well known from HST images, as well as the morphology of the main oval, which is known to remain stable with time (Grodent et al., 2003).

Also, the outputs resulting from the energy degradation model meet the observational constraints described in Table 2 of (Grodent et al., 2001). The value of $1020 \mathrm{~K}$ found for the $\mathrm{H}_{3}^{+}$temperature weighted by the $\mathrm{H}_{3}^{+}$emission profile (Table 2) is in the upper part of the observational range (700-1100 K ), although values up to $\sim 1500 \mathrm{~K}$ are suggested from Echelle spectra obtained with the Infrared Telescope Facility (IRTF) spectrometer (Stallard et al., 2002). The exospheric temperature of $3600 \mathrm{~K}$ obtained at the top of the 1-D model is significantly higher than values previously calculated, but no actual measurement of the exospheric temperature in the auroral region is available. Our Table 2 also shows that the $\mathrm{H}_{2}$ gas temperature weighted by the FUV auroral emission (305 K) is significantly lower than the $\mathrm{H}_{2}$ temperature weighted by the auroral emission above the methane homopause $(810 \mathrm{~K})$. The FUSE spectra studied here are characterized by temperatures in the order of $800 \mathrm{~K}$, which demonstrates that short-wavelength FUV auroral spectra probe the higher altitude part of the auroral UV emission and are not indicative of the bulk of the auroral energy deposition. The analysis of two GHRS high-resolution Echelle spectra in the 1216-1220 ̊ window in (Dols et al., 2000) pointed out that the 20-layer models used to fit the G140L low resolution spectra (from 1200 to $1700 \AA$ ) were too cold to reproduce the Echelle spectra (from 1216 to $1220 \AA$ ), taken close in time to the G140L spectra. At that time, a single Maxwellian was used to produce the models, with characteristic energies of 17 and $40 \mathrm{keV}$ for each model, respectively. A best fit was 
Table 2

Main characteristics of the multi-layer model used in this study (displayed in Fig. 16)

\begin{tabular}{lcrrrrr}
\hline & Maxwellian & & & & \\
\cline { 2 - 7 } & $\# 1$ & $\# 2$ & $\# 3$ & $\# 4$ & $\# 5$ & $\# 6$ \\
\hline$\Phi^{\mathrm{a}}$ & 50 & 30 & 8 & 80 & 100 & 10 \\
$E_{0}{ }^{\mathrm{b}}$ & 1 & 4 & 9 & 25 & 40 & 100
\end{tabular}

$\mathrm{H}_{2}$ temperature from the 1-D model, weighted by the UV volume emission rate $\mathrm{H}_{2}$ temperature above the methane layer, weighted by the volume emission rate $\mathrm{H}_{2}$ column above the altitude of $\tau_{\left(\mathrm{CH}_{4}\right)}=1$ $\mathrm{H}_{3}^{+}$temperature weighted by the $\mathrm{H}_{3}^{+}$brightness $1020 \mathrm{~K}$

a $\Phi$ is the energy flux of the Maxwellian components at the top of the atmosphere (in erg $\mathrm{cm}^{-2} \mathrm{~s}^{-1}$ ).

b $E_{0}$ is the characteristic energy (in keV).
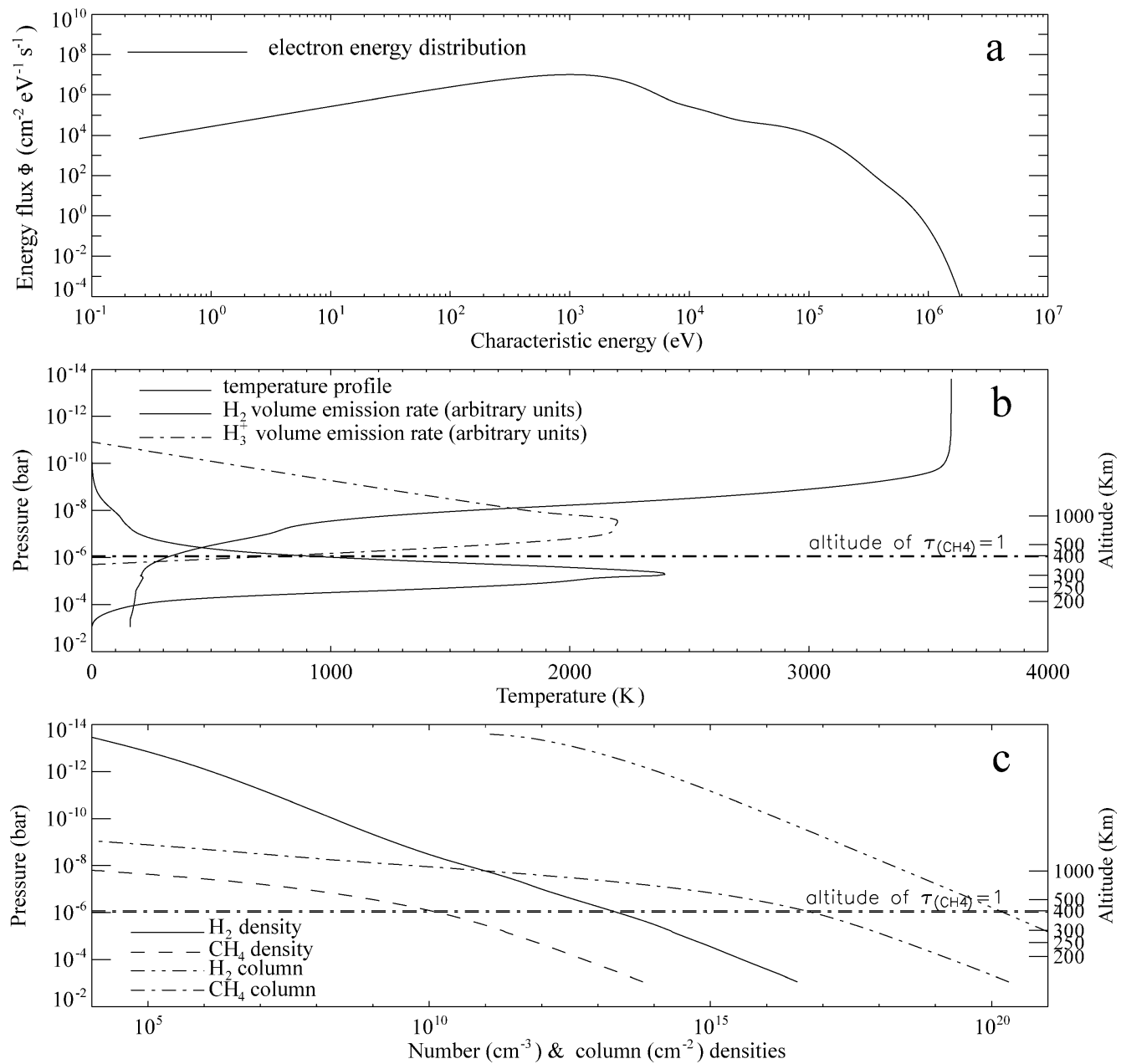

Fig. 16. Main characteristics of the best 1-D model atmosphere used to fit the three FUSE spectra and the HUT spectrum. (a) Energy distribution of the primary electrons precipitating at the top of the atmosphere. The flux and characteristic energies of the Maxwellian components that compose this distribution are displayed in Table 2. (b) Vertical thermal profile, $\log _{10}$ of the $\mathrm{H}_{2} \mathrm{FUV}$ volume emission rate and $\log _{10}$ of the $\mathrm{H}_{3}^{+}$volume emission rate as a function of pressure/altitude. It is seen that the short-wavelength FUV emission and the bulk of the $\mathrm{H}_{3}^{+}$emission probe approximately the same regions of the atmosphere. (c) $\mathrm{H}_{2}$ and $\mathrm{CH}_{4}$ densities and column densities as a function of pressure/altitude. The mean value of the methane cross-section between 900 and $1200 \AA$ is used to define the altitude of $\tau_{\left(\mathrm{CH}_{4}\right)}=1$ (marked up with a horizontal dash-dot line).

obtained with a single layer model at $600 \mathrm{~K}$, suggesting that the emission above the homopause needed to be higher in order to raise the mean temperature of the multi-layer model.

\subsection{Analysis of the HUT auroral spectrum}

A detailed comparison between single-layer models and the HUT jovian auroral spectrum can be found in Wolven 

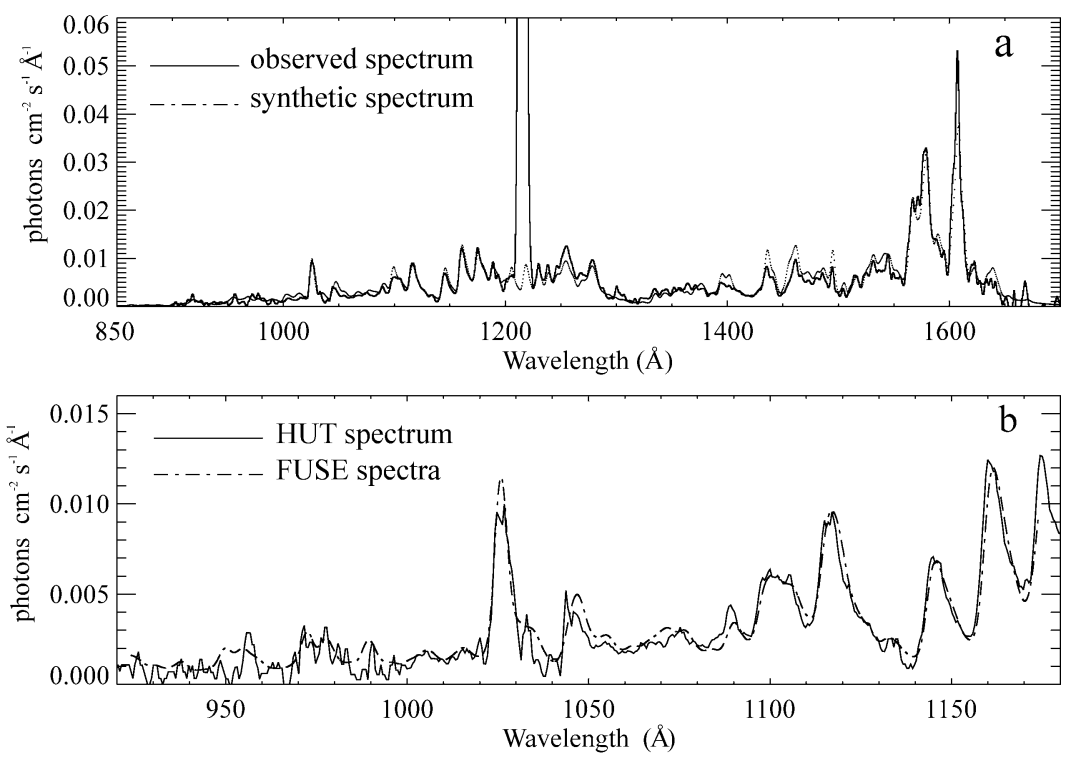

Fig. 17. (a) Comparison between the HUT auroral spectrum and the multi-layer model. This self-consistent model can reproduce the absorption by hydrocarbons and the $\mathrm{H}_{2}$ self-absorption. (b) Comparison between the EUV and short-wavelength FUV portion of the HUT spectrum and the FUSE spectra that were put together, degraded at the resolution of HUT, and scaled to the HUT brightness. The two spectra are almost identical—the main differences are seen below $1000 \AA$ where the HUT signal to noise is very low.

and Feldman (1998). Their best fit spectrum was obtained with a $\mathrm{H}_{2}$ slant column of $3 \times 10^{20} \mathrm{~cm}^{-2}$, a temperature of $900 \mathrm{~K}$ and an overlying methane column of $2 \times 10^{16} \mathrm{~cm}^{-2}$. Applying our single-layer model with the same value for the methane column, we obtain a best fit spectrum for an $\mathrm{H}_{2}$ slant column of $5 \times 10^{20} \mathrm{~cm}^{-2}$ and a temperature of $800 \mathrm{~K}$, which are close to the values obtained by Wolven and Feldman (1998). The best 20-layer model we used to fit the FUSE data was also applied to the HUT spectrum. The fit is shown in Fig. 17a, where the complete FUV and EUV domains were taken into account in the fitting procedure. The ratio between the unabsorbed part and absorbed part (by methane) of the spectrum is well reproduced, which indicates that the modeled depth of the emission peak is correct. Self-absorption is also well reproduced, as demonstrated by the correct intensities of the features at 1050 and $1100 \AA$. As was the case for the FUSE LiF2a spectrum, the 20-layer model overestimates the intensity of the lines connecting to $v^{\prime \prime}=2$ near $1100 \AA$ (cf. Fig. 13). The color ratio of the model is slightly too large and since the model is slightly too absorbed by methane, the fit routine tends to raise the synthetic spectrum in order to minimize the $\chi^{2}$, as if the model did not include sufficient self-absorption near $1100 \AA$ A. Single-layer models were not able to solve this problem, whatever the $\mathrm{H}_{2}$ column, Tem or Tsa we used. At this point, it should be noted that each single Maxwellian component used in the 1-D model to generate the best synthetic spectrum leads to an auroral emission peaking below the $1 \times 10^{-9}$ bar level. Consequently, no additional discrete low-energy component is needed to fit the EUV and short-wavelength FUV portions of the spectra. This conclusion is at variance with Ajello et al. (2001) who analyzed EUV + FUV auroral spectra from the Galileo EUVS spectrometer and from HUT. They applied a coupled two-stream model and synthetic spectral generator to the observed spectra and found that the observed spectra displayed excess emission in the EUV and short-wavelength FUV portion in comparison with their best synthetic spectrum. An optically thin flux of electrons (with characteristic energy of $27 \mathrm{eV}$ ) was included in the input of the two-stream model to best fit the data, leading to the conclusion that the aurora was composed of two sources: a deep aurora resulting from 5-100 keV electrons and a high-altitude aurora resulting from $20-200 \mathrm{eV}$ electrons, near the exobase. The observational constraints provided by the HUT spectrum do not impose the addition of a discrete soft electron component to fit the data. Therefore, the energy distribution presented here is likely to be more appropriate to specify the characteristics of the primary electrons precipitating at the top of the jovian atmosphere. The multi-Maxwellian energy distribution we used contains soft electrons: the total energy in the range $0-200 \mathrm{eV}$ is $\sim 1.4 \%$ of the total energy precipitating at the top of the atmosphere. The main effect of the lowenergy electrons $(<200 \mathrm{eV})$ is to warm up the atmosphere. Their contribution to the $\mathrm{H}_{2}$ volume emission rate is low, as seen in Fig. 16b: there is no secondary emission peak near the exobase.

\section{Conclusions}

The high spectral resolution of the FUSE spectra makes it possible to resolve individual rotational lines belonging to several Rydberg states of the $\mathrm{H}_{2}$ molecules. Numerical simulations show that the three spectral windows analyzed in this study are dominated by transitions connecting to $v^{\prime \prime}=1,2$, and 3 which saturate for self absorption at dif- 
ferent overlying $\mathrm{H}_{2}$ columns. Results from synthetic spectra show that the effective $\mathrm{H}_{2}$ temperature is on the order of $800 \mathrm{~K}$, a value higher than the $\sim 500 \mathrm{~K}$ found from earlier analysis of jovian spectra longward of $1400 \AA$, in the region where self absorption is negligible. The emission in the short-wavelength FUV spectral window is located just above the $\mathrm{CH}_{4}$ homopause and corresponds to vertical $\mathrm{H}_{2}$ columns on the order $1 \times 10^{20} \mathrm{~cm}^{-2}$, that is, to pressure levels close to $1 \mu \mathrm{bar}$.

The combination of the FUSE high-resolution spectrum and the HUT spectrum confirms these findings and shows that the bulk of the auroral energy deposition takes place near or below the homopause. This scenario accounts in a self-consistent way for the FUV color ratio measured with HUT and the amount of self absorption observed in both EUV spectra. One key conclusion of this study is that a distribution of relatively hard $(>1 \mathrm{keV})$ incident auroral electrons can fit all spectral characteristics of the FUV-EUV observations available so far. No additional discrete soft electron component is necessary to fit the short-wavelength FUV segment of the $\mathrm{H}_{2}$ jovian auroral spectrum. It should be stressed that the effective $\mathrm{H}_{2}$ FUV temperature $(300 \mathrm{~K}$ in the unabsorbed region) and the $\mathrm{H}_{3}^{+}$effective temperature $(1000 \mathrm{~K})$ are in the range of previous thermospheric temperature measurement (see the discussion by Grodent et al., 2001, private communication, 2001).

Short-wavelength FUV spectral observations probe regions above the hydrocarbon homopause and provide no direct observations concerning the region located below the altitude of $\tau \sim 1$ for self-absorption. They complement the long-wavelength FUV observations which reflect conditions prevailing near the emission peak (unabsorbed spectral region) or immediately below the hydrocarbon homopause (absorbed region). The $\mathrm{H}_{2}$ temperatures obtained in this study are an independent confirmation of the high temperatures in the auroral region derived from analysis of $\mathrm{H}_{3}^{+}$ spectra.

\section{Acknowledgments}

This work is based on data obtained for the Guaranteed Time Team by the NASA-CNES-CSA FUSE mission operated by the Johns Hopkins University. Financial support to US participants was provided by NASA contract NAS532985. J.-C.G. and D.G. acknowledge support from the Belgian Fund for Scientific Research (FNRS). The PRODEX program of ESA provided financial support for this research to the University of Liège. French participants are supported by CNES.

\section{References}

Abgrall, H., Roueff, E., Launay, F., Roncin, J.Y., Subtil, J.L., 1993a. Table of the Lyman band system of molecular hydrogen. Astron. Astrophys. Suppl. 101, 273-321.
Abgrall, H., Roueff, E., Launay, F., Roncin, J.Y., Subtil, J.L., 1993b. Table of the Werner band system of molecular hydrogen. Astron. Astrophys. Suppl. 101, 323-362.

Abgrall, H., Roueff, E., Launay, F., Roncin, J.Y., 1994. The B-Prime and D band systems of molecular hydrogen. Can. J. Phys. 72, 856-865.

Abgrall, H., Roueff, E., Drira, I., 2000. Total transition probability and spontaneous radiative dissociation of $B, C, B^{\prime}$ and $D$ states of molecular hydrogen. Astron. Astrophys. Suppl. 141, 297-300.

Abgrall, H., Roueff, E., Liu, X.M., Shemansky, D.E., 2004. Electron impact emission spectrum of $\mathrm{H}_{2}$ : the $B^{\prime \prime 1} \Sigma_{u}^{+}$and $D^{\prime 1} \Pi_{u}-X^{1} \Sigma_{g}^{+}$band system. J. Phys. B. Submitted for publication.

Ajello, J.M., 13 colleagues, 1998. Simultaneous extreme ultraviolet and far ultraviolet observations of Jupiter aurora from the Galileo Orbiter. J. Geophys. Res. 103, 20125-20148.

Ajello, J.M., Shemansky, D.E., Pryor, W.R., Stewart, A.I., Simmons, K.E., Majeed, T., Waite, J.H., Gladstone, G.R., Grodent, D., 2001. Spectroscopic evidence for high-altitude aurora at Jupiter from Galileo Extreme Ultraviolet Spectrometer and Hopkins Ultraviolet Telescope Observations. Icarus 152, 151-171.

Broadfoot, A.L., 16 colleagues, 1979. Extreme ultraviolet observations from Voyager 1 encounter with Jupiter. Science 204, 979-982.

Clarke, J.T., Ben Jaffel, L., Vidal-Madjar, A., Gladstone, G.R., Waite Jr., J.H., Prangé, R., Gérard, J.C., Ajello, J., James, G., 1994. Hubble Space Telescope Goddard high-resolution spectrograph $\mathrm{H}_{2}$ rotational spectra of Jupiter's aurora. Astrophys. J. 430, L73-L76.

Clarke, J.T., 20 colleagues, 1996. Far-ultraviolet imaging of Jupiter's aurora and the Io footprint. Science 274, 404-409.

Dziczek, D., Ajello, J.M., James, G.K., Hansen, D.L., 2000. A study of the cascade contribution to the $\mathrm{H}_{2}$ Lyman band system from electron impact. Phys. Rev. A 61, 64702-1-64702-4.

Feldman, P.D., McGrath, M.A., Moos, H.W., Durrance, S.T., Strobel, D.F., Davidsen, A.F., 1993. The spectrum of the jovian dayglow observed at $3 \AA$ resolution with the Hopkins Ultraviolet Telescope. Astrophys. J. 406, 279-284.

Gérard, J.-C., Singh, V., 1982. A model of energetic electrons and EUV emission in the jovian and saturnian atmospheres and implications. J. Geophys. Res. 87, 4525-4532.

Gérard, J.C., Grodent, D., Dols, V., Waite, J.H., 1998. The longitudinal variation of the color ratio of the jovian ultraviolet aurora: a geometric effect? Geophys. Res. Lett. 25, 1601-1604.

Gérard, J.C., Gustin, J., Grodent, D., Delamere, P., Clarke, J.T., 2002. Excitation of the FUV Io tail on Jupiter: characterization of the electron precipitation. J. Geophys. Res. 107 (A11), 1394-1401.

Gladstone, G.R., Allen, M., Yung, Y.L., 1996. Hydrocarbon photochemistry in the upper atmosphere of Jupiter. Icarus 119, 1-52.

Grodent, D., Waite Jr., J.H., Gérard, J.C., 2001. A self-consistent model of the jovian auroral thermal structure. J. Geophys. Res. 106, 1293312952.

Grodent, D., Clarke, J.T., Waite Jr., J.H., Kim, J., Cowley, S.W.H., 2003. Jupiter's main auroral oval observed with HST-STIS. J. Geophys. Res. 108, 1389-1405.

Gustin, J., Grodent, D., Gérard, J.C., Clarke, J.T., 2002. Spatially resolved far ultraviolet spectroscopy of the jovian aurora. Icarus 156, 91-103.

Harris, W., Clarke, J.T., McGrath, M.A., Ballester, G.E., 1996. Analysis of jovian auroral H Ly- $\alpha$ emission (1981-1991). Icarus 124, 350-365.

Jonin, C., Liu, X., Ajello, J.M., James, G.K., Abgrall, H., 2000. Highresolution electron-impact spectrum of $\mathrm{H}_{2}$. I. Cross sections and predissociation yields. Astrophys. J. Suppl. 129, 247-266.

Liu, X., Shemansky, D.E., Ahmed, S.M., James, G.K., Ajello, J.M., 1998. Electron-impact excitation and emission cross sections of the $\mathrm{H}_{2}$ Lyman and Werner systems. J. Geophys. Res. 103, 26739-26758.

Moos, H.W., Cash, W.C., Cowie, L.L., Davidsen, A.F., Dupree, A.K., Feldman, P.D., Friedman, S.D., Green, J.C., Green, R.F., Gry, C., Hutchings, J.B., Jenkins, E.B., Linsky, J.L., Malina, R.F., Michalitsianos, A.G., Savage, B.D., Shull, J.M., Siegmund, O.H.W., Snow, T.P., Son- 
neborn, G., Vidal-Madjar, A., Willis, A.J., Woodgate, B.E., York, D.G., Ake, T.B., Andersson, B., Andrews, J.P., Barkhouser, R.H., Bianchi, L., Blair, W.P., Brownsberger, K.R., Cha, A.N., Chayer, P., Conard, S.J., Fullerton, A.W., Gaines, G.A., Grange, R., Gummin, M.A., Hebrard, G., Kriss, G.A., Kruk, J.W., Mark, D., McCarthy, D.K., Morbey, C.L., Murowinski, R., Murphy, E.M., Oegerle, W.R., Ohl, R.G., Oliveira, C., Osterman, S.N., Sahnow, D.J., Saisse, M., Sembach, K.R., Weaver, H.A., Welsh, B.Y., Wilkinson, E., Zheng, W., 2000. Overview of the Far Ultraviolet Spectroscopic Explorer Mission. Astrophys. J. 538, L1-L6.

Reinhold, E., Hogervorst, W., Ubachs, W., Wolniewicz, L., 1999. Experimental and theoretical investigation of the $H \bar{H}^{1} \Sigma_{g}^{+}$state in $\mathrm{H}_{2}, \mathrm{D}_{2}$ and $\mathrm{HD}$, and the $B^{\prime \prime} \bar{B}^{1} \Sigma_{u}^{+}$state in HD. Phys. Rev. A 60, 1258-1270.

Sahnow, D.J., Moos, H.W., Ake, T.B., Andersen, J., Andersson, B., Andre, M., Artis, D., Berman, A.F., Blair, W.P., Brownsberger, K.R., Calvani, H.M., Chayer, P., Conard, S.J., Feldman, P.D., Friedman, S.D., Fullerton, A.W., Gaines, G.A., Gawne, W.C., Green, J.C., Gummin, M.A., Jennings, T.B., Joyce, J.B., Kaiser, M.E., Kruk, J.W., Lindler, D.J., Massa, D., Murphy, E.M., Oegerle, W.R., Ohl, R.G., Roberts, B.A., Romelfanger, M.L., Roth, K.C., Sankrit, R., Sembach, K.R., Shelton, R.L., Siegmund, O.H.W., Silva, C.J., Sonneborn, G., Vaclavik, S.R., Weaver, H.A., Wilkinson, E., 2000. On-orbit performance of the Far Ultraviolet Spectroscopic Explorer Satellite. Astrophys. J. 538, L7-L11.

Shemansky, D.E., Ajello, J.M., Hall, D.T., 1985. Electron impact excitation of $\mathrm{H}_{2}$ : Rydberg band systems and the benchmark dissociative cross section for H Lyman alpha. Astrophys. J. 296, 765-773.
Spielfiedel, A., 2003. Ab initio calculation of electronic transition moments for singlet excited states of the $\mathrm{H}_{2}$ molecule. J. Mol. Spectrosc. 217, $162-172$.

Stallard, T., Miller, S., Millward, G., Joseph, R.D., 2002. On the dynamics of the jovian ionosphere and thermosphere. II. The measurement of $\mathrm{H}_{3}^{+}$ vibrational temperature, column density and total emission. Icarus 156 , 498-514.

Trafton, L.M., Gérard, J.C., Munhoven, G., Waite, J.H., 1994. Highresolution of Jupiter northern auroral ultraviolet emission with the Hubble Space Telescope. Astrophys. J. 421, 816-827.

Trafton, L.M., Dols, V., Gérard, J.C., Waite, J.H., Gladstone, R.G., Munhoven, G., 1998. HST spectra of the jovian ultraviolet aurora: search for heavy ion precipitation. Astrophys. J. 507, 955-967.

Vasavada, A.R., Bouchez, A.H., Ingersoll, A.P., Little, B., Anger, C.D., the Galileo SSI Team, 1999. Jupiter's visible aurora and Io footprint. J. Geophys. Res. 104, 27133-27142.

Waite Jr., J.H., Cravens, T.E., Kozyra, J.U., Nagy, A.F., Atreya, S.K., Chen, R.H., 1983. Electron precipitation and related aeronomy of the jovian thermosphere and ionosphere. J. Geophys. Res. 88, 6143-6163.

Wolven, B.C., Feldman, P.D., 1998. Self-absorption by vibrationally excited $\mathrm{H}_{2}$ in the Astro-2 Hopkins Ultraviolet Telescope spectrum of the jovian aurora. Geophys. Res. Lett. 25, 1537-1540.

Yung, Y.L., Gladstone, G.R., Chang, K.M., Ajello, J.M., Srivastava, S.K., 1982. $\mathrm{H}_{2}$ fluorescence spectrum from 1200 to $1700 \AA$ by electron impact: laboratory study and application to jovian aurora. Astrophys. J. 254, L65-L69. 